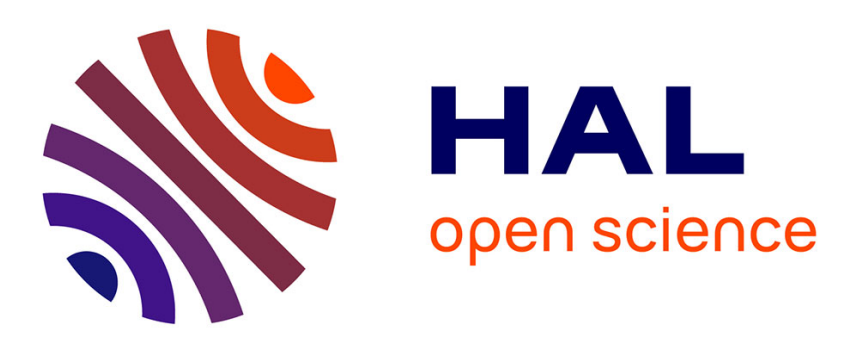

\title{
Analytical Analysis of Bayesian Cramer-Rao Bound for Dynamical Rayleigh Channel Complex Gains Estimation in OFDM System
}

\author{
Hussein Hijazi, Laurent Ros
}

\section{> To cite this version:}

Hussein Hijazi, Laurent Ros. Analytical Analysis of Bayesian Cramer-Rao Bound for Dynamical Rayleigh Channel Complex Gains Estimation in OFDM System. IEEE Transactions on Signal Processing, 2009, 57 (5), pp.1889. 10.1109/TSP.2009.2014264 . hal-00368687

\section{HAL Id: hal-00368687 \\ https://hal.science/hal-00368687}

Submitted on 17 Mar 2009

HAL is a multi-disciplinary open access archive for the deposit and dissemination of scientific research documents, whether they are published or not. The documents may come from teaching and research institutions in France or abroad, or from public or private research centers.
L'archive ouverte pluridisciplinaire HAL, est destinée au dépôt et à la diffusion de documents scientifiques de niveau recherche, publiés ou non, émanant des établissements d'enseignement et de recherche français ou étrangers, des laboratoires publics ou privés. 


\title{
Analytical Analysis of Bayesian Cramer-Rao Bound for Dynamical Rayleigh Channel Complex Gains Estimation in OFDM System
}

\author{
Hussein Hijazi and Laurent Ros \\ GIPSA-lab, Department Image Signal, BP 46 - 38402 Saint Martin d'Hères - FRANCE
}

\begin{abstract}
In this paper, we consider the Bayesian Cramer-Rao bound (BCRB) for the dynamical estimation of multi-path Rayleigh channel complex gains in data-aided (DA) and non-data-aided (NDA) OFDM systems. This bound is derived in an on-line and off-line scenarios for time-invariant and time-varying complex gains within one OFDM symbol, assuming the availability of prior information. In NDA context, whereas this true $\mathrm{BCRB}$ is hard to evaluate, we present a closed-form expression of a $\mathrm{BCRB}$, i.e., the Asymptotic BCRB (ABCRB) or the Modified BCRB (MBCRB). We discuss, based on the theoretical and simulation results, the interest of using some past and future observations in terms of Doppler spread for the complex gains estimation.
\end{abstract}

\section{Index Terms}

Bayesian Cramer-Rao Bound, OFDM, Rayleigh complex gains.

\section{INTRODUCTION}

In the case of wideband Orthogonal Frequency Division Multiplexing (OFDM) mobile communication systems, dynamic channel estimation [10] [13] is a fundamental function, because the radio channel is

Copyright (c) 2008 IEEE. Personal use of this material is permitted. However, permission to use this material for any other purposes must be obtained from the IEEE by sending a request to pubs-permissions@ieee.org.

Part of this work was presented in IEEE ISWCS, Reykjavik, Iceland, October 2008 [1]. The authors are with the GIPSA lab. (ex LIS lab.), Image and Signal Department, Grenoble, France (e-mail: hussein.hijazi@gipsa-lab.inpg.fr, laurent.ros@gipsalab.inpg.fr). 
frequency selective and time-varying [19]. Channel estimation can be summarized to estimate certain physical propagation parameters, such as multi-path delays and multi-path complex gains. Working with the time-domain model, Tsatsanis and Giannakis propose in [6] a linear algorithm for estimating timevarying FIR systems where each time-varying tap coefficient can be described as a linear combination of a finite number of basis functions. Moreover, Borah and Hart propose in [7] a method for estimating the impulse response of a frequency-selective fading channel using a polynomial model of the time-varying channel taps. Recently, the basis expansion model (BEM) was introduced to approximate OFDM channel variations. Firstly, for slow fading assumptions, [8] used a polynomial basis function model for the channel response in a time-frequency window, whereas [9] modeled the correlated discrete-time fading channel using a Karhunen-Loeve(KL) orthogonal expansion. For fast time-varying channels, many existing works resort to estimating the equivalent discrete-time channel taps, which are modeled by the BEM [10] [11]. In MC-CDMA system, Zemen and Mecklenbrauker propose in [12] a low-complexity channel estimator for a time-variant frequency-selective channel using discrete prolate spheroidal (DPS) sequences. However, in Radio-Frequencies transmission, the delays are quasi invariant over several OFDM symbols but the complex gains may change significantly, even within one OFDM symbol. Exploiting the channel nature and assuming the availability of delay information, a lot of methods estimate the time-variations of the multi-path complex gains in OFDM [14] [2] [3] [4] [5] and CDMA [15] [16] systems.

In this context the question arises of the ultimate accuracy that can be achieved in channel estimation operations. Establishing bounds to such an accuracy is an important goal since it provides benchmarks for evaluating the performance of channel estimators. Tools to approach this problem are available from the parameters estimation theory [20] [27] in the form of Cramer-Rao Bounds (CRBs), which give fundamental lower limits to the variance of any parameter estimator. A Modified CRB (MCRB), easier to evaluate than the Standard CRB (SCRB), has been introduced in [21] [22]. The MCRB proves useful when, in addition to the parameter to be estimated, the observed data also depend on other unwanted parameters. However, the problem of deriving CRBs suited to time-varying parameters has been addressed throughout the Bayesian context [20] [23]. More recently, the authors propose in [24] a general framework for deriving analytical expression of on-line Bayesian CRB (BCRB). In [25], the authors introduce a new asymptotic bound for the mono-carrier phase estimation problem, namely the Asymptotic Bayesian CRB (ABCRB), in NDA scenario. This bound is closer to the classical BCRB than the Modified BCRB (MBCRB) and is easier to evaluate than BCRB.

In this contribution we investigate the BCRB related to the estimation of Rayleigh channel complex gains with Jakes spectrum for OFDM systems. We distinguish two channel variation cases: "time-varying" 
and "time-invariant" complex gains within one OFDM symbol. Explicit expressions of the BCRB and its variants, MBCRB and ABCRB, are provided in NDA and DA contexts and, in on-line and off-line scenarios, assuming the availability of prior information.

This paper is organized as follows: Section II sets the system model, whereas Section III recalls the general BCRB and the modified MBCRB. Section IV derives the BCRB, the MBCRB and the ABCRB for "time-varying" and "time-invariant" multi-path complex gains estimation. Section V illustrates and interpretes different results. Finally, our conclusions are presented in Section VI.

The notations adopted are as follows: Upper (lower) bold face letters denote matrices (column vectors). $[\mathbf{x}]_{k}$ denotes the $k$ th element of the vector $\mathbf{x}$, and $[\mathbf{X}]_{k, m}$ denotes the $[k, m]$ th element of the matrix $\mathbf{X}$. We will use the matlab notation $\mathbf{X}_{\left[k_{1}: k_{2}, m_{1}: m_{2}\right]}$ to extract a submatrix within $\mathbf{X}$ from row $k_{1}$ to row $k_{2}$ and from column $m_{1}$ to column $m_{2} . \mathbf{I}_{N}$ is a $N \times N$ identity matrix and $\mathbf{0}_{N}$ is a $N \times N$ matrix of zeros. $\operatorname{diag}\{\mathbf{x}\}$ is a diagonal matrix with $\mathbf{x}$ on its main diagonal, $\operatorname{diag}\{\mathbf{X}\}$ is a vector whose elements are the elements of the main diagonal of $\mathbf{X}$ and blkdiag $\{\mathbf{X}, \mathbf{Y}\}$ is a block diagonal matrix with the matrices $\mathbf{X}$ and $\mathbf{Y}$ on its main diagonal. The superscripts $(\cdot)^{T}$ and $(\cdot)^{H}$ stand respectively for transpose and Hermitian operators. $|\cdot|$, and $\operatorname{Tr}(\cdot)$ are respectively the determinant and trace operations. $\operatorname{Re}(\cdot), \operatorname{Im}(\cdot)$ and $(\cdot)^{*}$ are respectively the real part, imaginary part and conjugate of a complex number or matrix. $\mathrm{E}_{x, y}[\cdot]$ is the expectation over $x$ and $y, J_{0}(\cdot)$ is the zeroth-order Bessel function of the first kind and $\delta_{k, m}$ is the Kronecker symbol. $\nabla_{\mathbf{x}}$ and $\Delta_{\mathbf{y}}^{\mathbf{x}}$ represent the first and the second-order partial derivatives operator i.e., $\nabla_{\mathbf{x}}=\left[\frac{\partial}{\partial x_{1}}, \ldots, \frac{\partial}{\partial x_{N}}\right]^{T}$ and $\Delta_{\mathbf{y}}^{\mathbf{x}}=\nabla_{\mathbf{y}}^{*} \nabla_{\mathbf{x}}^{T}$.

\section{OFDM SYSTEM AND CHANNEL MODELS}

\section{A. OFDM System Model}

Consider an OFDM system with $\mathrm{N}$ sub-carriers, and a cyclic prefix length $N_{g}$. The duration of an OFDM symbol is $T=v T_{s}$, where $T_{s}$ is the sampling time and $v=N+N_{g}$. Let $\mathbf{x}_{(n)}=\left[x_{(n)}\left[-\frac{N}{2}\right], x_{(n)}\left[-\frac{N}{2}+\right.\right.$ $\left.1], \ldots, x_{(n)}\left[\frac{N}{2}-1\right]\right]^{T}$ be the $n$th transmitted OFDM symbol, where $\left\{x_{(n)}[b]\right\}$ are normalized symbols (i.e., $\mathrm{E}\left[x_{(n)}[b] x_{(n)}[b]^{*}\right]=1$ ). After transmission over a multi-path Rayleigh channel, the $n$th received OFDM symbol $\mathbf{y}_{(n)}=\left[y_{(n)}\left[-\frac{N}{2}\right], y_{(n)}\left[-\frac{N}{2}+1\right], \ldots, y_{(n)}\left[\frac{N}{2}-1\right]\right]^{T}$ is given by [3] [5]:

$$
\mathbf{y}_{(n)}=\mathbf{H}_{(n)} \mathbf{x}_{(n)}+\mathbf{w}_{(n)}
$$


where $\mathbf{w}_{(n)}=\left[w_{(n)}\left[-\frac{N}{2}\right], w_{(n)}\left[-\frac{N}{2}+1\right], \ldots, w_{(n)}\left[\frac{N}{2}-1\right]\right]^{T}$ is a white complex Gaussian noise vector with covariance matrix $\sigma^{2} \mathbf{I}_{N}$ and $\mathbf{H}_{(n)}$ is a $N \times N$ channel matrix with elements given by:

$$
\left[\mathbf{H}_{(n)}\right]_{k, m}=\frac{1}{N} \sum_{l=1}^{L}\left[e^{-j 2 \pi\left(\frac{m-1}{N}-\frac{1}{2}\right) \tau_{l}} \sum_{q=0}^{N-1} \alpha_{l}^{(n)}\left(q T_{s}\right) e^{j 2 \pi \frac{m-k}{N} q}\right]
$$

where $L$ is the total number of propagation paths, $\alpha_{l}$ is the lth complex gain of variance $\sigma_{\alpha_{l}}^{2}$ and $\tau_{l} \times T_{s}$ is the $l$ th delay $\left(\tau_{l}\right.$ is not necessarily an integer, but $\tau_{L}<N_{g}$ ). The L individual elements of $\left\{\alpha_{l}^{(n)}\left(q T_{s}\right)=\alpha_{l}\left(q T_{s}+n T\right)\right\}$ are uncorrellated with respect to each other. They are wide-sense stationary (WSS), narrow-band complex Gaussian processes, with the so-called Jakes' power spectrum of maximum Doppler frequency $f_{d}$, i.e., $\mathrm{E}\left[\alpha_{l}\left(q_{1} T_{s}\right) \alpha_{l}^{*}\left(q_{2} T_{s}\right)\right]=\sigma_{\alpha_{l}}^{2} J_{0}\left(2 \pi f_{d} T_{s}\left(q_{1}-q_{2}\right)\right)$ [17]. The average energy of the channel is normalized to one, i.e., $\sum_{l=1}^{L} \sigma_{\alpha_{l}}^{2}=1$.

\section{B. Complex Gain Model}

Since the number of samples to estimate $L v$ is greater than the number of observation equations $N$, it is not efficient to estimate the multi-path complex gains time-variation, using directly the observation model in (1). Therefore, we represent the time-variation of the complex gains by a more compact model. In the literature, the basis expansion model (BEM) was introduced to approximate OFDM channel variations. The BEM methods [10] are Karhunen-Loeve BEM (KL-BEM), prolate spheroidal BEM (PS-BEM), complex-exponential BEM (CE-BEM) and polynomial BEM (P-BEM). In [13], a piece-wise linear method is used to approximate the equivalent discrete-time channel taps. In [4] [3], the authors show that the time-variation of Rayleigh channel complex gain, within $N_{c}$ OFDM symbols, can be approximated by a polynomial model of $N_{c}$ coefficients, choosen according to the Doppler spread $f_{d} T$.

In this section, we show that, whatever $f_{d} T \leq 0.5$, each Rayleigh channel complex gain $\boldsymbol{\alpha}_{l}^{(n)}=$ $\left[\alpha_{l}^{(n)}\left(-N_{g} T_{s}\right), \ldots, \alpha_{l}^{(n)}\left((N-1) T_{s}\right)\right]^{T}$ can be modeled as a polynomial time-variation of $N_{c} \leq 5$ coefficients (i.e., a $\left(N_{c}-1\right)$ degree polynomial), within one OFDM symbol.

The optimal polynomial $\boldsymbol{\alpha}_{\mathbf{p o l}}^{(n)}$, which is least-squares fitted (linear and polynomial regression) [18] to $\boldsymbol{\alpha}_{l}^{(n)}$, and its $N_{c}$ coefficients $\mathbf{c}_{l}^{(n)}=\left[c_{1, l}^{(n)}, \ldots, c_{N_{c}, l}^{(n)}\right]^{T}$ are given by:

$$
\begin{aligned}
\boldsymbol{\alpha}_{\mathbf{p o} \mathbf{l}_{l}}^{(n)} & =\mathbf{Q}^{T} \mathbf{c}_{l}^{(n)}=\mathbf{S} \boldsymbol{\alpha}_{l}^{(n)} \\
\mathbf{c}_{l}^{(n)} & =\left(\mathbf{Q Q} \mathbf{Q}^{T}\right)^{-1} \mathbf{Q} \boldsymbol{\alpha}_{l}^{(n)}
\end{aligned}
$$

where $\mathbf{Q}$ is a $N_{c} \times v$ matrix of elements $[\mathbf{Q}]_{k, m}=\left(m-N_{g}-1\right)^{(k-1)}$ and $\mathbf{S}=\mathbf{Q}^{T}\left(\mathbf{Q} \mathbf{Q}^{T}\right)^{-1} \mathbf{Q}$ is a $v \times v$ matrix. It provides the MMSE approximation for all polynomials containing $N_{c}$ coefficients, given by:

$$
\operatorname{MMSE}_{l}^{(0)}=\frac{1}{v} \operatorname{Tr}\left(\mathbf{M M S E}_{l}^{(0)}\right)
$$


where

$$
\mathbf{M M S E}_{l}^{(p)}=\mathrm{E}\left[\boldsymbol{\xi}_{l}^{(n)} \boldsymbol{\xi}_{l}^{(n-p)^{H}}\right]=\left(\mathbf{I}_{v}-\mathbf{S}\right) \mathbf{R}_{\boldsymbol{\alpha}_{l}}^{(p)}\left(\mathbf{I}_{v}-\mathbf{S}^{T}\right)
$$

with $\boldsymbol{\xi}_{l}^{(n)}=\boldsymbol{\alpha}_{l}^{(n)}-\boldsymbol{\alpha}_{\mathbf{p o l}}^{(n)}{ }_{l}$ is the model error and $\mathbf{R}_{\boldsymbol{\alpha}_{l}}^{(p)}=\mathrm{E}\left[\boldsymbol{\alpha}_{l}^{(n)} \boldsymbol{\alpha}_{l}^{(n-p)^{H}}\right]$ is the $v \times v$ correlation matrix of $\boldsymbol{\alpha}_{l}^{(n)}$ with elements given by:

$$
\left[\mathbf{R}_{\boldsymbol{\alpha}_{l}}^{(p)}\right]_{k, m}=\sigma_{\alpha_{l}}^{2} J_{0}\left(2 \pi f_{d} T_{s}(k-m+p v)\right)
$$

Fig. 1 gives the MMSE in terms of $f_{d} T$ for different value of $N_{c}$. As can be seen, for $f_{d} T \leq 0.5$ and $N_{c}=5$, we have MMSE $<4 \cdot 10^{-7}$. This proves that, for high values of $f_{d} T, \boldsymbol{\alpha}_{l}^{(n)}$ can be represented by a polynomial model of $N_{c} \leq 5$ coefficients. Moreover, for $f_{d} T \leq 0.001$ and $N_{c}=1$, we have MMSE $<4 \cdot 10^{-7}$. This means that, for low values of $f_{d} T$, the complex gains are time-invariant within one OFDM symbol.

$\mathbf{c}_{l}^{(n)}$ are correlated complex Gaussian variables with zero-means and correlation matrix given by:

$$
\mathbf{R}_{\mathbf{c}_{l}}^{(p)}=\mathrm{E}\left[\mathbf{c}_{l}^{(n)} \mathbf{c}_{l}^{(n-p)^{H}}\right]=\left(\mathbf{Q} \mathbf{Q}^{T}\right)^{-1} \mathbf{Q} \mathbf{R}_{\boldsymbol{\alpha}_{l}}^{(p)} \mathbf{Q}^{T}\left(\mathbf{Q} \mathbf{Q}^{T}\right)^{-1}
$$

Fig. 2 shows the average (over $L=6$ paths) variance of the first three coefficients of the $N_{c}=5$ coefficients. We notice that the variance decreases very quickly in terms of number of coefficients. This means that the last coefficients are very small. Hence, it is very difficult to find an estimator that can give a good estimation of the small coefficients in presence of noise. In the sequel, we will study the performance of the coefficients estimator in terms of $N_{c}$ and $f_{d} T$.

Under this polynomial modeling, the observation model in (1) for the $n$th OFDM symbol can be rewritten as:

$$
\mathbf{y}_{(n)}=\mathcal{K}_{(n)} \mathbf{c}_{(n)}+\boldsymbol{\epsilon}_{(n)}+\mathbf{w}_{(n)}
$$

where $\mathbf{c}_{(n)}=\left[\mathbf{c}_{1}^{(n)^{T}}, \ldots, \mathbf{c}_{L}^{(n)^{T}}\right]^{T}$ is a $L N_{c} \times 1$ vector, $\mathcal{K}_{(n)}=\frac{1}{N}\left[\mathbf{Z}_{1}^{(n)}, \ldots, \mathbf{Z}_{L}^{(n)}\right]$ is a $N \times L N_{c}$ matrix and $\mathbf{Z}_{l}^{(n)}=\left[\mathbf{M}_{1} \operatorname{diag}\left\{\mathbf{x}_{(n)}\right\} \mathbf{f}_{l}, \ldots, \mathbf{M}_{N_{c}} \operatorname{diag}\left\{\mathbf{x}_{(n)}\right\} \mathbf{f}_{l}\right]$ is a $N \times N_{c}$ matrix, where $\mathbf{f}_{l}$ is the $l$ th column of the $N \times L$ Fourier matrix $\mathbf{F}$ and $\mathbf{M}_{d}$ is a $N \times N$ matrix given by:

$$
[\mathbf{F}]_{k, l}=e^{-j 2 \pi\left(\frac{k-1}{N}-\frac{1}{2}\right) \tau_{l}}, \quad\left[\mathbf{M}_{d}\right]_{k, m}=\sum_{q=0}^{N-1} q^{d-1} e^{j 2 \pi \frac{m-k}{N} q}
$$

The second component in (8), $\boldsymbol{\epsilon}_{(n)}$, represents the polynomial approximation error in the observation model which is given by:

$$
\boldsymbol{\epsilon}_{(n)}=\mathbf{H}_{\boldsymbol{\xi}_{(n)}} \mathbf{x}_{(n)}
$$


where $\mathbf{H}_{\boldsymbol{\xi}_{(n)}}$ is a $N \times N$ matrix with elements given by:

$$
\left[\mathbf{H}_{\boldsymbol{\xi}_{(n)}}\right]_{k, m}=\frac{1}{N} \sum_{l=1}^{L}\left[e^{-j 2 \pi\left(\frac{m-1}{N}-\frac{1}{2}\right) \tau_{l}} \sum_{q=0}^{N-1} \xi_{l}^{(n)}\left(q T_{s}\right) e^{j 2 \pi \frac{m-k}{N} q}\right]
$$

It sould be noted that if the complex gains are time-invariant within one OFDM symbol $\left(i . e ., \alpha_{l}^{(n)}\left(-N_{g} T_{s}\right)=\right.$ $\left.\ldots=\alpha_{l}^{(n)}\left((N-1) T_{s}\right)=c_{1, l}^{(n)}\right)$ then, $\mathbf{H}_{(n)}$ is a diagonal matrix, $N_{c}=1, \mathcal{K}_{(n)}=\operatorname{diag}\left\{\mathbf{x}_{(n)}\right\} \mathbf{F}$ and $\mathbf{R}_{\mathbf{c}_{l}}^{(p)}=\sigma_{\alpha_{l}}^{2} J_{0}\left(2 \pi f_{d} T p\right)$.

\section{CRAMER-RAO BOUndS (CRBS)}

In this section, we present the family of Cramer-Rao Bounds (CRBs). The CRBs provide a lower bound on the Mean Square Error (MSE) achievable by any unbiased estimator. We give the general expression of the Bayesian Cramer-Rao Bound (BCRB) and its Modified Version (MBCRB). The BCRB is particularly suited for problems where the parameter to be estimated is assumed to be random with availablity of the a priori information. Let $\hat{\mathbf{c}}(\mathbf{y})$ denotes an unbiased estimator of $\mathbf{c}$ using the set of measurements $\mathbf{y}$. The estimation of $\mathbf{c}$ can be considered following two main scenarios off-line and on-line. In the off-line scenario, the receiver waits until the whole observation frame, i.e., $\mathbf{y}=\left[\mathbf{y}_{(1)}{ }^{T}, \ldots, \mathbf{y}_{(K)}\right]^{T}$, has been received in order to estimate $\left.\mathbf{c}=\left[\mathbf{c}_{(1)}{ }^{T}, \ldots, \mathbf{c}_{(K)}\right]^{T}\right]^{T}$. In the on-line scenario, the receiver estimates $\mathbf{c}_{(n)}$ based on the current and previous observations only, i.e., $\mathbf{y}=\left[\mathbf{y}_{(1)}{ }^{T}, \ldots, \mathbf{y}_{(n)}{ }^{T}\right]^{T}$. In the sequel, the BCRB will be considered within the context of both the off-line and the on-line scenarios. The BCRB has been proposed in [20] such that:

$$
\mathrm{E}_{\mathbf{y}, \mathbf{c}}\left[(\hat{\mathbf{c}}(\mathbf{y})-\mathbf{c})(\hat{\mathbf{c}}(\mathbf{y})-\mathbf{c})^{H}\right] \geq \mathbf{B C R B}(\mathbf{c})
$$

The $\mathrm{BCRB}^{1}$ is the inverse of the Bayesian Information Matrix (BIM), which can be written as:

$$
\mathbf{B}=\mathrm{E}_{\mathbf{c}}[\mathbf{F i}(\mathbf{c})]+\mathrm{E}_{\mathbf{c}}\left[-\Delta_{\mathbf{c}}^{\mathbf{c}} \ln (p(\mathbf{c}))\right]
$$

where $p(\mathbf{c})$ is the prior distribution and $\mathbf{F i}(\mathbf{c})$ is the Fisher Information Matrix (FIM) defined as:

$$
\mathbf{F i}(\mathbf{c})=\mathrm{E}_{\mathbf{y} \mid \mathbf{c}}\left[-\Delta_{\mathbf{c}}^{\mathbf{c}} \ln (p(\mathbf{y} \mid \mathbf{c}))\right]
$$

where $p(\mathbf{y} \mid \mathbf{c})$ is the conditional probability density function of $\mathbf{y}$ given $\mathbf{c}$. Unfortunately, in most cases of NDA context, the computation of $\mathbf{F i}(\mathbf{c})$ is generally quite tedious because the $p(\mathbf{y} \mid \mathbf{c})$ cannot be carried out analytically due to the nuisance parameters $\mathbf{x}=\left[\mathbf{x}_{(1)}{ }^{T}, \ldots, \mathbf{x}_{(K)}{ }^{T}\right]^{T}$, which are OFDM symbols in our

\footnotetext{
${ }^{1}$ We recall that, for a deterministic parameter, Standard Cramer-Rao Bound (SCRB) would be directly the inverse of the Fisher Information Matrix (FIM).
} 
case. In order to circumvent this problem, a Modified BCRB (MBCRB) has been proposed in [23]. This MBCRB is the inverse of the following information matrix:

$$
\mathbf{C}=\mathrm{E}_{\mathbf{c}}[\mathbf{G}(\mathbf{c})]+\mathrm{E}_{\mathbf{c}}\left[-\Delta_{\mathbf{c}}^{\mathbf{c}} \ln (p(\mathbf{c}))\right]
$$

where $\mathbf{G}(\mathbf{c})$ is the modified FIM defined as:

$$
\mathbf{G}(\mathbf{c})=\mathrm{E}_{\mathbf{x}} \mathrm{E}_{\mathbf{y} \mid \mathbf{x}, \mathbf{c}}\left[-\Delta_{\mathbf{c}}^{\mathbf{c}} \ln (p(\mathbf{y} \mid \mathbf{x}, \mathbf{c}))\right]
$$

In our objective, we are interested in the estimation of the random complex gains $\boldsymbol{\alpha}=\left[\boldsymbol{\alpha}_{(1)}^{T}, \ldots, \boldsymbol{\alpha}_{(K)}^{T}\right]^{T}$, where $\boldsymbol{\alpha}_{(n)}=\left[\boldsymbol{\alpha}_{1}^{(n)^{T}}, \ldots, \boldsymbol{\alpha}_{L}^{(n)^{T}}\right]^{T} . \boldsymbol{\alpha}$ is related to $\mathbf{c}$ as:

$$
\alpha=\mathcal{Q} \mathbf{c}+\xi
$$

where $\mathcal{Q}=\operatorname{blkdiag}\left\{\mathbf{Q}^{T}, \ldots, \mathbf{Q}^{T}\right\}$ is a $K L v \times K L N_{c}$ matrix and $\boldsymbol{\xi}=\left[\boldsymbol{\xi}_{(1)}^{T}, \ldots, \boldsymbol{\xi}_{(K)}^{T}\right]^{T}$ with $\boldsymbol{\xi}_{(n)}=$ $\left[\boldsymbol{\xi}_{1}^{(n)^{T}}, \ldots, \boldsymbol{\xi}_{L}^{(n)^{T}}\right]^{T}$. Hence the estimation of $\boldsymbol{\alpha}$ is given by: $\hat{\boldsymbol{\alpha}}=\mathcal{Q} \hat{\mathbf{c}}$. By neglecting the cross-covariance terms between the errors $\boldsymbol{\alpha}_{\text {pol }}-\hat{\alpha}$ and $\boldsymbol{\xi}$, we can write:

$$
\begin{aligned}
& \mathrm{E}\left[(\hat{\boldsymbol{\alpha}}-\boldsymbol{\alpha})(\hat{\boldsymbol{\alpha}}-\boldsymbol{\alpha})^{H}\right]= \\
& \mathrm{E}\left[\left(\hat{\boldsymbol{\alpha}}-\boldsymbol{\alpha}_{\mathbf{p o l}}\right)\left(\hat{\boldsymbol{\alpha}}-\boldsymbol{\alpha}_{\mathbf{p o l}}\right)^{H}\right]+\mathrm{E}\left[\boldsymbol{\xi} \boldsymbol{\xi}^{H}\right]
\end{aligned}
$$

where $\boldsymbol{\alpha}_{\text {pol }}=\mathcal{Q}$ c. So, using the transformation of parameters properties defined in [27], we obtain the BCRB for the estimation of $\alpha$ from the BCRB for $\mathbf{c}$ as:

$$
\begin{aligned}
\operatorname{BCRB}(\boldsymbol{\alpha}) & =\left(\nabla_{\mathbf{c}} \boldsymbol{\alpha}_{\mathbf{p o l}}\right) \mathbf{B C R B}(\mathbf{c})\left(\nabla_{\mathbf{c}} \boldsymbol{\alpha}_{\mathbf{p o l}}^{T}\right)+\mathrm{E}\left[\boldsymbol{\xi} \xi^{H}\right] \\
& =\mathcal{Q} \mathbf{B C R B}(\mathbf{c}) \mathcal{Q}^{T}+\mathbf{M M S E}
\end{aligned}
$$

where the $K L v \times K L v$ matrix MMSE is given by:

$$
\operatorname{MMSE}_{\left[i(l, p), i\left(l, p^{\prime}\right)\right]}=\mathbf{M M S E}_{l}^{\left(p-p^{\prime}\right)} \text { for } l \in[1, L] p, p^{\prime} \in[0, K-1]
$$

with $i(l, p)=1+(l-1) v+p L v: l v+p L v$ and $\mathbf{M M S E}_{l}^{(p)}$ is the correlation matrix of the model error $\xi_{l}^{(n)}$ defined in (5). Notice that there are zero matrices between the block matrices $\mathbf{M M S E}_{l}^{(p)}$ since the L complex gains are uncorrellated. For $K=L=2$, MMSE is given by:

$$
\mathbf{M M S E}=\left[\begin{array}{cccc}
\mathbf{M M S E}_{1}^{(0)} & \mathbf{0}_{v} & \mathbf{M M S E}_{1}^{(-1)} & \mathbf{0}_{v} \\
\mathbf{0}_{v} & \mathbf{M M S E}_{2}^{(0)} & \mathbf{0}_{v} & \mathbf{M M S E}_{2}^{(-1)} \\
\mathbf{M M S E}_{1}^{(1)} & \mathbf{0}_{v} & \mathbf{M M E}_{1}^{(0)} & \mathbf{0}_{v} \\
\mathbf{0}_{v} & \mathbf{M M S E}_{2}^{(1)} & \mathbf{0}_{v} & \mathbf{M M S E}_{2}^{(0)}
\end{array}\right]
$$


The computation of the off-line BCRB [25] associated to the estimation of $\boldsymbol{\alpha}_{(n)}$ is given by:

$$
\operatorname{BCRB}\left(\boldsymbol{\alpha}_{(n)}\right)_{o f f l i n e}=\operatorname{Tr}\left(\mathbf{B C R B}(\boldsymbol{\alpha})_{[i(n), i(n)]}\right)
$$

where the sequence of indices $i(n)=1+(n-1) L v: n L v$ with $n \in[1, K]$. The on-line BCRB [25] associated to the observation vector $\mathbf{y}=\left[\mathbf{y}_{(1)}{ }^{T}, \ldots, \mathbf{y}_{(K)}{ }^{T}\right]^{T}$ is given by:

$$
\operatorname{BCRB}\left(\boldsymbol{\alpha}_{(K)}\right)_{\text {online }}=\operatorname{Tr}\left(\mathbf{B C R B}(\boldsymbol{\alpha})_{[i(K), i(K)]}\right)
$$

The definitions in (19), (21) and (22) will stand for the closed form of BCRB, i.e., MBCRB and ABCRB.

\section{BCRB FOR Polynomial CoEfFicients Estimation}

In this section, we present a closed-form expression for a BCRB related to the estimation of the polynomial coefficients $\mathbf{c}_{(n)}$ of the multi-path complex gains in NDA OFDM systems. This bound is derived for time-varying and time-invariant complex gains within one OFDM symbol. In DA context, we deduce the computation of the true BCRB from the computation of the MBCRB in NDA.

\section{A. BCRB for Time-varying Complex Gains}

1) Non-Data-Aided (NDA) Context:

Computation of $\mathbf{E}_{\mathbf{c}}[\mathbf{F i}(\mathbf{c})]$ : The observation model is presented in (8). Using the whiteness of the noise $\mathbf{w}=\left[\mathbf{w}_{(1)}^{T}, \ldots, \mathbf{w}_{(K)}\right]^{T}$ and the independence of the transmitted OFDM symbols $\mathbf{x}$, we then obtain that:

$$
\Delta_{\mathbf{c}}^{\mathbf{c}} \ln (p(\mathbf{y} \mid \mathbf{c}))=\sum_{n=1}^{K} \Delta_{\mathbf{c}}^{\mathbf{c}} \ln \left(p\left(\mathbf{y}_{(n)} \mid \mathbf{c}_{(n)}\right)\right)
$$

It is important to note that each term of the summation (23) is a $K L N_{c} \times K L N_{c}$ block diagonal matrix with only one nonzero $L N_{c} \times L N_{c}$ block matrix, namely:

$$
\Delta_{\mathbf{c}}^{\mathbf{c}} \ln \left(p\left(\mathbf{y}_{(n)} \mid \mathbf{c}_{(n)}\right)\right)_{\left[i^{\prime}(n), i^{\prime}(n)\right]}=\Delta_{\mathbf{c}_{(n)}}^{\mathbf{c}_{(n)}} \ln \left(p\left(\mathbf{y}_{(n)} \mid \mathbf{c}_{(n)}\right)\right)
$$

where $i^{\prime}(n)=1+(n-1) L N_{c}: n L N_{c}$ with $n \in[1, K]$. As a direct consequence, $\Delta_{\mathbf{c}}^{\mathbf{c}} \ln (p(\mathbf{y} \mid \mathbf{c}))$ is a block diagonal matrix with the $n$th diagonal block given by (24). Moreover, because of the circularity of the observation noise, the expectation of (24) with respect to $\mathbf{y}_{(n)}$ and $\mathbf{c}_{(n)}$ does not depend on $\mathbf{c}_{(n)}$. We then obtain:

$$
\mathrm{E}_{\mathbf{c}}[\mathbf{F i}(\mathbf{c})]=\operatorname{blkdiag}\{\mathbf{J}, \mathbf{J}, \ldots, \mathbf{J}\}
$$

where $\mathbf{J}$ is a $L N_{c} \times L N_{c}$ matrix defined as:

$$
\mathbf{J}=\mathrm{E}_{\mathbf{y}, \mathbf{c}}\left[-\Delta_{\mathbf{c}_{(n)}}^{\mathbf{c}_{(n)}} \ln \left(p\left(\mathbf{y}_{(n)} \mid \mathbf{c}_{(n)}\right)\right)\right]
$$


The log-likelihood function in (26) can be expanded as:

$$
\ln \left(p\left(\mathbf{y}_{(n)} \mid \mathbf{c}_{(n)}\right)\right)=\ln \left(\sum_{\mathbf{x}_{(n)}} p\left(\mathbf{y}_{(n)} \mid \mathbf{x}_{(n)}, \mathbf{c}_{(n)}\right) p\left(\mathbf{x}_{(n)}\right)\right)
$$

In order to simplify the computation of $p\left(\mathbf{y}_{(n)} \mid \mathbf{x}_{(n)}, \mathbf{c}_{(n)}\right)$, we assume that the vector $\boldsymbol{\xi}_{(n)}$ for given $\mathbf{c}_{(n)}$ remains complex Gaussian vector with zero mean. Hence, the vector $\mathbf{y}_{(n)}$ for given $\mathbf{x}_{(n)}$ and $\mathbf{c}_{(n)}$ is a complex Gaussian vector with mean vector $\mathbf{m}_{(n)}=\mathcal{K}_{(n)} \mathbf{c}_{(n)}$ and covariance matrix $\boldsymbol{\Omega}=\mathcal{R}+\sigma^{2} \mathbf{I}_{N}$, where $\mathcal{R}$ is the $N \times N$ correlation matrix of $\boldsymbol{\epsilon}_{(n)}$ given by (see Appendix A):

$$
\mathcal{R}=\mathrm{E}_{\boldsymbol{\xi}_{(n)}, \mathbf{x}_{(n)}}\left[\boldsymbol{\epsilon}_{(n)} \boldsymbol{\epsilon}_{(n)}^{H}\right]=\frac{\beta}{N} \boldsymbol{\Lambda} \operatorname{diag}\{\operatorname{diag}\{\boldsymbol{\Gamma}\}\} \boldsymbol{\Lambda}^{H}
$$

where $\beta=\sum_{l=1}^{L} \sigma_{\alpha_{l}}^{2}$ is the total channel energy, $\boldsymbol{\Lambda}$ and $\boldsymbol{\Gamma}$ are two $N \times N$ matrices defined by:

$$
\begin{aligned}
{[\boldsymbol{\Lambda}]_{k, m} } & =e^{-j 2 \pi \frac{k}{N}(m-1)} \\
\boldsymbol{\Gamma} & =\frac{1}{\sigma_{\alpha_{l}}^{2}} \mathbf{M M S E}_{l}^{(0)}{ }_{\left[N_{g}+1: v, N_{g}+1: v\right]}
\end{aligned}
$$

Thus, $p\left(\mathbf{y}_{(n)} \mid \mathbf{x}_{(n)}, \mathbf{c}_{(n)}\right)$ is defined as:

$$
p\left(\mathbf{y}_{(n)} \mid \mathbf{x}_{(n)}, \mathbf{c}_{(n)}\right)=\frac{1}{|\pi \boldsymbol{\Omega}|} e^{-\left(\mathbf{y}_{(n)}-\mathbf{m}_{(n)}\right)^{H} \boldsymbol{\Omega}^{-1}\left(\mathbf{y}_{(n)}-\mathbf{m}_{(n)}\right)}
$$

Since each element of the vector $\mathbf{m}_{(n)}$ depends on all components of $\mathbf{x}_{(n)}$ then, the computation of $\mathbf{J}$ is a demanding task. Hence, we resort to compute the MBCRB. Following the same reasoning as before, we have:

$$
\mathrm{E}_{\mathbf{c}}[\mathbf{G}(\mathbf{c})]=\operatorname{blkdiag}\left\{\mathbf{J}_{m}, \mathbf{J}_{m}, \ldots, \mathbf{J}_{m}\right\}
$$

where $\mathbf{J}_{m}$ is a $L N_{c} \times L N_{c}$ matrix defined as:

$$
\mathbf{J}_{m}=\mathrm{E}_{\mathbf{y}, \mathbf{x}, \mathbf{c}}\left[-\Delta_{\mathbf{c}_{(n)}}^{\mathbf{c}_{(n)}} \ln \left(p\left(\mathbf{y}_{(n)} \mid \mathbf{x}_{(n)}, \mathbf{c}_{(n)}\right)\right)\right]
$$

By taking the second derivative of the natural logarithm $(l n)$ of (31) with respect to $\mathbf{c}_{(n)}$, we simply obtain that:

$$
\Delta_{\mathbf{c}_{(n)}}^{\mathbf{c}_{(n)}} \ln \left(p\left(\mathbf{y}_{(n)} \mid \mathbf{x}_{(n)}, \mathbf{c}_{(n)}\right)\right)=-\mathcal{K}_{(n)}^{H} \boldsymbol{\Omega}^{-1} \mathcal{K}_{(n)}
$$

Consequently, we obtain that (see Appendix B):

$$
\mathbf{J}_{m}=\mathrm{E}_{\mathbf{x}}\left[\mathcal{K}_{(n)}^{H} \boldsymbol{\Omega}^{-1} \mathcal{K}_{(n)}\right]=\frac{1}{N^{2}} \mathcal{F}^{H} \mathcal{M} \mathcal{F}
$$


where $\mathcal{M}$ and $\mathcal{F}$ are a $N N_{c} \times N N_{c}$ and a $N N_{c} \times L N_{c}$ matrices, respectively, defined as:

$$
\begin{aligned}
\mathcal{M} & =\left[\begin{array}{ccc}
\mathcal{M}_{1,1} & \cdots & \mathcal{M}_{1, N_{c}} \\
\vdots & \ddots & \vdots \\
\mathcal{M}_{N_{c}, 1} & \cdots & \mathcal{M}_{N_{c}, N_{c}}
\end{array}\right] \\
\mathcal{F} & =\left[\begin{array}{lll}
\mathcal{F}_{1} & \cdots & \mathcal{F}_{L}
\end{array}\right]
\end{aligned}
$$

where $\mathcal{M}_{d, d^{\prime}}$ and $\mathcal{F}_{l}$ are a $N \times N$ and a $N N_{c} \times N_{c}$ matrices, respectively, defined as:

$$
\begin{aligned}
\mathcal{M}_{d, d^{\prime}} & =\operatorname{diag}\left\{\operatorname{diag}\left\{\mathbf{M}_{d}^{H} \boldsymbol{\Omega}^{-1} \mathbf{M}_{d^{\prime}}\right\}\right\} \\
\mathcal{F}_{l} & =\operatorname{blkdiag}\left\{\mathbf{f}_{l}, \mathbf{f}_{l}, \ldots, \mathbf{f}_{l}\right\}
\end{aligned}
$$

Computation of $\mathbf{E}_{\mathbf{c}}\left[-\Delta_{\mathbf{c}}^{\mathbf{c}} \ln (p(\mathbf{c}))\right]: \mathbf{c}$ is a complex Gaussian vector with zero mean and covariance matrix $\mathbf{R}_{\mathbf{c}}$ of size $K L N_{c} \times K L N_{c}$ defined as:

$$
\mathbf{R}_{\mathbf{c}\left[i^{\prime}(l, p), i^{\prime}\left(l, p^{\prime}\right)\right]}=\mathbf{R}_{\mathbf{c}_{l}}^{\left(p-p^{\prime}\right)} \text { for } l \in[1, L] p, p^{\prime} \in[0, K-1]
$$

where $i^{\prime}(l, p)=1+(l-1) N_{c}+p L N_{c}: l N_{c}+p L N_{c}$ and $\mathbf{R}_{\mathbf{c}_{l}}^{(p)}$ is the correlation matrix of $\mathbf{c}_{l}^{(n)}$ defined in (7). Thus, the probability density function $p(\mathbf{c})$ is defined as:

$$
p(\mathbf{c})=\frac{1}{\left|\pi \mathbf{R}_{\mathbf{c}}\right|} e^{-\mathbf{c}^{H} \mathbf{R}_{\mathbf{c}}^{-1} \mathbf{c}}
$$

Taking the second derivative of the natural logarithm $(l n)$ of (41) with respect to $\mathbf{c}$ and making the expectation over $\mathbf{c}$, we simply obtain that:

$$
\mathrm{E}_{\mathbf{c}}\left[-\Delta_{\mathbf{c}}^{\mathbf{c}} \ln (p(\mathbf{c}))\right]=\mathbf{R}_{\mathbf{c}}^{-1}
$$

The MBCRB for the estimation of $\mathbf{c}$ in NDA context is given by:

$$
\operatorname{MBCRB}(\mathbf{c})=\left(\operatorname{blkdiag}\left\{\mathbf{J}_{m}, \mathbf{J}_{m}, \ldots, \mathbf{J}_{m}\right\}+\mathbf{R}_{\mathbf{c}}^{-1}\right)^{-1}
$$

Notice that the MBCRB is usually looser than the BCRB. As in (19), the MBCRB for the estimation of $\alpha$ is given by:

$$
\operatorname{MBCRB}(\boldsymbol{\alpha})=\mathcal{Q} \operatorname{MBCRB}(\mathbf{c}) \mathcal{Q}^{T}+\operatorname{MMSE}
$$




\section{2) Data-Aided (DA) Context:}

In data-aided (DA) context, the transmitted data symbols $\mathbf{x}_{(n)}$ are known at the receiver and then no averaging over the data is required. Hence, the matrix $\mathbf{J}$ is computed like $\mathbf{J}_{m}$, but without averaging over the data symbols $\mathbf{x}_{(n)}$, and consequently it depends on the $n$th transmitted OFDM symbol. Thus, $\mathbf{J}_{(n)}$ is given by:

$$
\mathbf{J}_{(n)}=\mathcal{K}_{(n)}^{H} \boldsymbol{\Omega}_{(n)}^{-1} \mathcal{K}_{(n)}=\frac{1}{N^{2}} \mathcal{F}_{(n)}^{H} \mathcal{M}_{(n)} \mathcal{F}_{(n)}
$$

where $\boldsymbol{\Omega}_{(n)}=\boldsymbol{\mathcal { R }}_{(n)}+\sigma^{2} \mathbf{I}_{N}$ with $\boldsymbol{\mathcal { R }}_{(n)}$ is given by (see Appendix A):

$$
\boldsymbol{\mathcal { R }}_{(n)}=\mathrm{E}_{\boldsymbol{\xi}_{(n)}}\left[\boldsymbol{\epsilon}_{(n)} \boldsymbol{\epsilon}_{(n)}^{H}\right]=\frac{1}{N^{2}} \boldsymbol{\Lambda}\left(\boldsymbol{\Gamma} \bullet \mathcal{Z}_{(n)}\right) \boldsymbol{\Lambda}^{H}
$$

where $\bullet$ is the element-by-element product operator and $\mathcal{Z}_{(n)}$ is a $N \times N$ matrix given by:

$$
\mathcal{Z}_{(n)}=\boldsymbol{\Lambda}^{H} \operatorname{diag}\left\{\mathbf{x}_{(n)}\right\} \mathbf{F} \mathbf{D} \mathbf{F}^{H} \operatorname{diag}\left\{\mathbf{x}_{(n)}^{H}\right\} \mathbf{\Lambda}
$$

with $\mathbf{D}=\operatorname{diag}\left\{\sigma_{\alpha_{1}}^{2}, \ldots, \sigma_{\alpha_{L}}^{2}\right\}$. The matrix $\mathcal{M}_{(n)}$ is computed like $\mathcal{M}$ but by replacing $\boldsymbol{\Omega}$ in equation (38) by $\boldsymbol{\Omega}_{(n)}$ and, the matrix $\mathcal{F}_{(n)}$ is computed like $\mathcal{F}$ but by replacing $\mathbf{f}_{l}$ in equation (39) by $\operatorname{diag}\left\{\mathbf{x}_{(n)}\right\} \mathbf{f}_{l}$.

The BCRB for the estimation of $\mathbf{c}$ in DA context is given by:

$$
\operatorname{BCRB}(\mathbf{c})=\left(\operatorname{blkdiag}\left\{\mathbf{J}_{(1)}, \mathbf{J}_{(2)}, \ldots, \mathbf{J}_{(K)}\right\}+\mathbf{R}_{\mathbf{c}}^{-1}\right)^{-1}
$$

and consequently the BCRB for the estimation of $\boldsymbol{\alpha}$ as (19). It should be noted that BCRB for the estimation of $\boldsymbol{\alpha}$ in DA context depends on the transmitted data sequence $\mathbf{x}$.

\section{B. BCRB for Time-invariant Complex Gains}

In this paragraph, we derive an analytical expression of the BCRB and MBCRB associated to the estimation of time-invariant Rayleigh complex gains within one OFDM symbol in NDA context. In such case, we have: $\mathcal{K}_{(n)}=\operatorname{diag}\left\{\mathbf{x}_{(n)}\right\} \mathbf{F}, N_{c}=1, \mathbf{R}_{\mathbf{c}_{l}}^{(p)}=\sigma_{\alpha_{l}}^{2} J_{0}\left(2 \pi f_{d} T p\right), \mathbf{B C R B}(\boldsymbol{\alpha})=\mathbf{B C R B}(\mathbf{c})$ and $\boldsymbol{\Omega}=\sigma^{2} \mathbf{I}_{N}$. Hence, substituting this result in (35), we obtain $\mathbf{J}_{m}=\frac{1}{\sigma^{2}} \mathbf{F}^{H} \mathbf{F}$, and consequently MBCRB as (45).

In this case, each element of the vector $\mathbf{m}_{(n)}=\operatorname{diag}\left\{\mathbf{x}_{(n)}\right\} \mathbf{F} \mathbf{c}_{(n)}$ depends on only one element of $\mathbf{x}_{(n)}$ then, the computation of $\mathbf{J}$ is possible. It should be noted that, in NDA context, the BCRB depends on the modulation scheme of the OFDM symbols. Therefore, in the sequel, we evaluate $\mathbf{J}$ for 4QAM OFDM symbols. Hence, using the Gaussian nature of the noise and the equiprobability of the normalized QAM 
symbols, we find that (see Appendix C):

$$
\ln \left(p\left(\mathbf{y}_{(n)} \mid \mathbf{c}_{(n)}\right)\right)=\ln \left[\frac{1}{\left|\pi \sigma^{2} \mathbf{I}_{N}\right|} e^{-\frac{1}{\sigma^{2}}\left(\mathbf{y}_{(n)}^{H} \mathbf{y}_{(n)}+\mathbf{c}_{(n)}^{H} \mathbf{F}^{H} \mathbf{F} \mathbf{c}_{(n)}\right)} \prod_{k=1}^{N} \cosh \left(\frac{\sqrt{2}}{\sigma^{2}} \operatorname{Re}\left(a_{n}(k)\right)\right) \cosh \left(\frac{\sqrt{2}}{\sigma^{2}} \operatorname{Im}\left(a_{n}(k)\right)\right)\right]
$$

where $a_{n}(k)=\left[\mathbf{y}_{(n)}\right]_{k}^{*} \mathbf{g}_{k}^{T} \mathbf{c}_{(n)}$ and $\mathbf{g}_{k}^{T}$ is the $k$ th row of the matrix $\mathbf{F}$. The result of the second derivative of (49) with respect to $\mathbf{c}_{(n)}$ is given by (see Appendix C):

$$
\begin{gathered}
\Delta_{\mathbf{c}_{(n)}}^{\mathbf{c}_{(n)}} \ln \left(p\left(\mathbf{y}_{(n)} \mid \mathbf{c}_{(n)}\right)\right)=-\frac{1}{\sigma^{2}} \mathbf{F}^{H} \mathbf{F} \\
+\sum_{k=1}^{N}\left[\frac{1}{2 \sigma^{4}}\left[\mathbf{y}_{(n)}\right]_{k}\left[\mathbf{y}_{(n)}\right]_{k}^{*} \mathbf{g}_{k}^{*} \mathbf{g}_{k}^{T}\left(2-\tanh ^{2}\left(\frac{\sqrt{2}}{\sigma^{2}} \operatorname{Re}\left(a_{n}(k)\right)\right)-\tanh ^{2}\left(\frac{\sqrt{2}}{\sigma^{2}} \operatorname{Im}\left(a_{n}(k)\right)\right)\right)\right]
\end{gathered}
$$

In the general case, the expectation of (50) with respect to $\mathbf{y}_{(n)} \mid \mathbf{c}_{(n)}$ does not have any simple analytical solution. Hence, in practice, we have to resort to either numerical integration methods or some approximations. In the following, we present both the high-SNR and the low-SNR approximations of the BCRB, as defined in [25].

High-SNR BCRB Asymptote: Now, we investigate the BCRB behavior at high SNR. From the definition of the BIM (13), only the first term (i.e., $\mathrm{E}_{\mathbf{c}}[\mathbf{F i}(\mathbf{c})]$ ) depends on the SNR, which is fully characterized by $\mathbf{J}$. Hence, we focus on the behavior of $\mathbf{J}$. At high SNR (i.e., $\sigma^{2} \rightarrow 0$ ), the tanh-function in (50) can be approximated as: $\tanh \left(\frac{\sqrt{2}}{\sigma^{2}} x\right) \approx \operatorname{sgn}(x)$. Hence, we obtain the high-SNR asymptote of $\mathbf{J}$, which is:

$$
\mathbf{J}_{h}=\frac{1}{\sigma^{2}} \mathbf{F}^{H} \mathbf{F}
$$

This implies that $\mathbf{J}_{h}=\mathbf{J}_{m}$, hence the high-SNR asymptote of the BCRB is equal to the MBCRB. This corroborates the result derived by Moeneclaey [26] in the non-Bayesian case for a scalar parameter.

Low-SNR BCRB Asymptote: Now, we consider, the low-SNR asymptote of the BCRB in the NDA QAM context. Following the same reasoning as before, at low $\operatorname{SNR}\left(\right.$ i.e., $\left.\sigma^{2} \rightarrow+\infty\right)$, we have $\tanh (x) \approx$ $x$ arround $x=0$. Hence, we obtain:

$$
\Delta_{\mathbf{c}_{(n)}}^{\mathbf{c}_{(n)}} \ln \left(p\left(\mathbf{y}_{(n)} \mid \mathbf{c}_{(n)}\right)\right) \approx-\frac{1}{\sigma^{2}} \mathbf{F}^{H} \mathbf{F}+\sum_{k=1}^{N}\left[\frac{1}{\sigma^{8}}\left[\mathbf{y}_{(n)}\right]_{k}\left[\mathbf{y}_{(n)}\right]_{k}^{*} \mathbf{g}_{k}^{*} \mathbf{g}_{k}^{T}\left(\sigma^{4}-a_{n}(k) a_{n}^{*}(k)\right)\right]
$$

Substituting (52) in (26), we obtain the low-SNR asymptote of $\mathbf{J}$, which is (see Appendix D):

$$
\mathbf{J}_{l}=\left(\frac{\beta}{\sigma^{4}}+\frac{8 \beta^{2}}{\sigma^{6}}+\frac{6 \beta^{3}}{\sigma^{8}}\right) \mathbf{F}^{H} \mathbf{F}
$$

The Asymptotic BCRB (ABCRB) defined in [25] leads to a lower bound on the MSE. This ABCRB is given by:

$$
\operatorname{ABCRB}(\mathbf{c})=\left(\operatorname{blkdiag}\left\{\mathbf{J}_{m i n}, \ldots, \mathbf{J}_{m i n}\right\}+\mathbf{R}_{\mathbf{c}}^{-1}\right)^{-1}
$$


where $\mathbf{J}_{\min }=\min \left(v_{l}, v_{h}\right) \mathbf{F}^{H} \mathbf{F}, v_{l}=\frac{\beta}{\sigma^{4}}+\frac{8 \beta^{2}}{\sigma^{6}}+\frac{6 \beta^{3}}{\sigma^{8}}$ and $v_{h}=\frac{1}{\sigma^{2}}$. In Appendix E, we show that:

$$
\operatorname{MBCRB}(\mathbf{c}) \leq \operatorname{ABCRB}(\mathrm{c}) \leq \operatorname{BCRB}(\mathrm{c})
$$

This corroborates the result derived in [25] for a mono-carrier phase estimation problem.

Notice that the term $\mathcal{K}_{(n)}^{H} \boldsymbol{\Omega}^{-1} \mathcal{K}_{(n)}=\frac{1}{\sigma^{2}} \mathbf{F}^{H} \mathbf{F}$ does not depend on the transmitted data sequence $\mathbf{x}$. Hence, the FIM defined in (14) and the modified FIM defined in (16) are equals. So, in the case of "time-invariant", the true BCRB in data-aided (DA) context is equal to the MBCRB in non-data-aided (NDA) context.

\section{Discussion}

In this section, we bring to the fore the behavior of the previous bounds, namely the off-line and the on-line BCRBs (DA context), MBCRBs and ABCRBs (NDA context) for the complex gains estimation. A normalized 4QAM OFDM system, $N=128$ subcarriers, $N_{g}=\frac{N}{8}$ subcarriers is used (note that $\mathrm{SNR}=\frac{1}{\sigma^{2}}$ and $\left.(\mathrm{SNR}) d B=\left(\frac{E_{b}}{N_{0}}\right) d B+3 d B\right)$. The normalized channel model is Rayleigh with $L=6$ paths of parameters given in [2] [3] [5]. We consider two scenarios: "time-invariant" with $N_{c}=1$ and $f_{d} T=10^{-3}$, and "time-varying" with $2 \leq N_{c} \leq 5$ and $0.05 \leq f_{d} T \leq 0.5$. we remind that, in timeinvariant scenario, the BCRB in DA context and the MBCRB in NDA context are equals . It sould be noted that, in case of time-varying scenario and DA context, the BCRBs are computed with a transmitted data sequence generated by a Maximal-Length Sequences (MLS) generator [28] of 13 shift registers with a feedback polynomial $[20033]_{8}$ (octal representation).

Fig. 3 superimposes versus time index, the on-line and the off-line ABCRBs (NDA and time-invariant, $f_{d} T=0.001$ ) or BCRBs (DA and time-varying, $f_{d} T=0.1$ and $N_{c}=2$ ) for different block-observation lengths $K$ at $\mathrm{SNR}=10 d B$. In the off-line context, we can see that the best complex gains estimation is achieved at the midblock, whereas the estimates are likely to be poorer at the block border. This stems from the fact that in the center position of the polynomial coefficients vector $\mathbf{c}$ we have more adjacent (past or future) and strongly correlated variables than at the border of the vector $\mathbf{c}$. Concerning the online bound, at the beginning when the number of observations increases, the estimator takes into account more and more information and the estimation is improved; the bound thus decreases and converges to an asymptote. The estimation performance is then limited by the observation noise independently of the number of observations taken into account. However, in order to reach the asymptote, it is sufficient to use 10 past OFDM symbols for the slow channel $\left(f_{d} T=0.001\right)$ whereas 3 past OFDM symbols for the rapid channel $\left(f_{d} T=0.1\right)$. 
We now analyse the bound behavior versus the SNR over a block of $K=20$ and $K=1$. Fig. 4 superimposes the BCRBs evaluated over $10^{4}$ OFDM symbols Monte Carlo trials and the ABCRBs for NDA context and time-invariant scenario $\left(f_{d} T=0.001\right)$ in (a) and, the BCRBs for DA context and timevarying scenario $\left(f_{d} T=0.1\right)$ in (b). We can verify that $M B C R B \leq A B C R B \leq B C R B$ as proved in Appendix E. At low SNR, the on-line bound $(n=K=20)$ and the off-line bound $(n=10)$ coincide because the observation noise masks the a priori dynamic polynomial coefficients evolution. However, as SNR increases, the off-line bound decreases more than the on-line bound because the information provided by each observation $\mathbf{y}_{(n)}$ is preponderant over the a priori knowledge of $\mathbf{c}$. At high SNR, the MBCRB and the ABCRB are very close, as predicted by our theoretical analysis.

We now want to emphasize the benefit of using past symbols in time-invariant scenario. For this, we make a zoom on Fig. 4 (a) as shown in Fig. 5 (a) and we add as benchmark the BCRBs for a blockobservation length $K=1$ (as in Fig. 4 (b)). This benchmark corresponds to the case where the estimator uses only the current symbol instead of the current and past symbols. We can obviously measure the great potential gain obtained by taking into account the past information ( $K=20$ versus $K=1$ ). However, we can also measure, from Fig. 5 (a), the potential performance improvement for Data Aided (MBCRB $=\mathrm{BCRB})$ versus Non Data Aided (BRCB) contexts in time-invariant scenario. In time-varying scenario and NDA context, we can not evaluate the true BCRB even with Monte Carlo trials. So, we can only compare a lower closed-form of the BCRB in NDA context, the MBCRB, to the true BCRB in DA context as shown in Fig. 5 (b). We can even so notice a potential performance improvement for DA with respect to NDA in time-varying scenario.

We now study the bound behavior versus $N_{c}$ and SNR over a block of $K=10$, in case of time-varying. Fig. 6 gives the BCRBs for $f_{d} T=0.5$ in terms of SNR in (a) and $N_{c}$ in (b). We observe in (a) that, whatever SNR, the bound is not always decreasing in terms of $N_{c}$ and at high SNR, the bound converges to the MMSE (the model error). As we see in (b), for $\mathrm{SNR}=15 \mathrm{~dB}, 25 \mathrm{~dB}$ and $35 \mathrm{~dB}$, the minimum of the bound is obtained at $N_{c}=3,4$ and 5 polynomial coefficients, respectively. This is due to the last coefficients which will be poorly estimated in presence of noise. Indeed, they are negligible compared to the noise level as we have seen in Fig. 2. Hence, in order to have a good estimation of the complex gains time-variation, we have to choose $N_{c}$ according to SNR and $f_{d} T$. The Table I shows how to selecte $N_{c}$, for realistic values of SNR and different values of $f_{d} T$, such that the bound is minimal. For example if $f_{d} T=0.3$, we choose $N_{c}=3$ and 4 for SNR $\in[0 ; 29]$ and SNR $\in[29 ; 40]$, respectively. We can 
introduce a New BCRB (NBCRB) which is independant of $N_{c}$, defined as:

$$
\operatorname{NBCRB}(\boldsymbol{\alpha})=\min _{N_{c}}(\mathbf{B C R B}(\boldsymbol{\alpha}))
$$

where $\min _{N_{c}}(\cdot)$ is the minimum over $N_{c}$. This definition in (56) will stand for the ABCRB and the MBCRB in case of NDA.

We now analyse the bound behavior versus $f_{d} T$. Fig. 7 gives the NBCRB in DA context (time-invariant and time-varying) versus $f_{d} T$ for $\mathrm{SNR}=20 d B$ and $K=10$. We notice that the NBCRB increases in terms of $f_{d} T$. This is because the correlation between variables becomes stronger when $f_{d} T$ decreases. So, the estimation gain for slow channel variations is more significant.

\section{CONCLUSION}

In this contibution, we have derived an analytical expression of a BCRB for the estimation of timevarying and time-invariant Rayleigh channel complex gains within one OFDM symbol. In case of timevarying, we have introduced a New BCRB (NBCRB) and we have shown that a good estimation of the complex gains time variation can be obtained by choosing the number of polynomial coefficients according to the noise level and the Doppler spread. These bounds are useful when analyzing the performance of complex gains estimators in DA and NDA contexts and in on-line and off-line scenarios. Moreover, we have shown the benefit of using the past OFDM symbols in channel estimation process, whereas most methods use only the current symbol.

\section{APPENDIX A}

\section{EVALUATION OF THE CORRELATION MATRIX $\mathcal{R}$}

In this Appendix, we detail the calculus to obtain the expression of the correlation matrix $\mathcal{R}$ of the polynomial approximation error in the observation model, $\boldsymbol{\epsilon}_{(n)}=\mathbf{H}_{\boldsymbol{\xi}_{(n)}} \mathbf{x}_{(n)}$, in both context data-aided (DA) and non-data-aided (NDA). The $N \times N$ correlation matrix $\mathcal{R}$ is defined by:

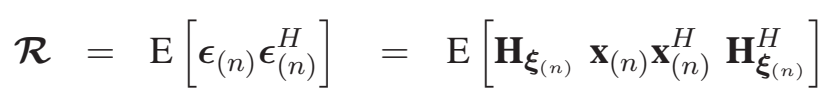

Hence, the elements of $\mathcal{R}$ are given by (57), shown at the top of the next page. since the elements $\left[\mathbf{H}_{\boldsymbol{\xi}_{(n)}}\right]_{k, m}$ and $\left[\mathbf{x}_{(n)}\right]_{k}$ are uncorrelated. Using (11), the first expectation in (57) can be calculated as (58), since the $\mathrm{L}$ different model errors $\left\{\xi\left[q T_{s}\right]\right\}$ are uncorrelated where $\boldsymbol{\Gamma}$ is defined by (30).

In NDA context, we have $\mathrm{E}\left[\left[\mathbf{x}_{(n)}\right]_{u_{1}}\left[\mathbf{x}_{(n)}\right]_{u_{2}}^{*}\right]=\delta_{u_{1}, u_{2}}$ since the unknown data symbols are uncorrelated and normalized, where $\delta_{k, m}$ is the Kronecker symbol. Hence, by using (58), the equation (57) becomes 


$$
\begin{gathered}
\left.[\mathcal{R}]_{k, m}=\mathrm{E}\left[\sum_{u_{1}=1}^{N} \sum_{u_{2}=1}^{N}\left[\mathbf{H}_{\boldsymbol{\xi}_{(n)}}\right]\right]_{k, u_{1}}\left[\mathbf{H}_{\boldsymbol{\xi}_{(n)}}\right]_{m, u_{2}}^{*}\left[\mathbf{x}_{(n)}\right]_{u_{1}}\left[\mathbf{x}_{(n)}\right]_{u_{2}}^{*}\right]=\sum_{u_{1}=1}^{N} \sum_{u_{2}=1}^{N} \mathrm{E}\left[\left[\mathbf{H}_{\boldsymbol{\xi}_{(n)}}\right]_{k, u_{1}}\left[\mathbf{H}_{\boldsymbol{\xi}_{(n)}}\right]_{m, u_{2}}^{*}\right] \mathrm{E}\left[\left[\mathbf{x}_{(n)}\right]_{u_{1}}\left[\mathbf{x}_{(n)}\right]_{u_{2}}^{*}\right] \\
\mathrm{E}\left[\left[\mathbf{H}_{\boldsymbol{\xi}_{(n)}}\right]_{k, u_{1}}\left[\mathbf{H}_{\boldsymbol{\xi}_{(n)}}\right]_{m, u_{2}}^{*}\right]=\frac{1}{N^{2}} \sum_{l=1}^{L} e^{j 2 \pi \frac{u_{2}-u_{1}}{N} \tau_{l}}\left[\sum_{q_{1}=0}^{N-1} \sum_{q_{2}=0}^{N-1} \mathrm{E}\left[\xi_{l}^{(n)}\left(q_{1} T_{s}\right) \xi_{l}^{(n)^{*}}\left(q_{2} T_{s}\right)\right] e^{j 2 \pi \frac{u_{1}-k}{N} q_{1}} e^{-j 2 \pi \frac{u_{2}-m}{N} q_{2}}\right] \\
=\frac{1}{N^{2}} \sum_{l=1}^{L} \sigma_{\alpha_{l}}^{2} e^{j 2 \pi \frac{u_{2}-u_{1}}{N} \tau_{l}}\left[\sum_{q_{1}=0}^{N-1} \sum_{q_{2}=0}^{N-1}[\boldsymbol{\Gamma}]_{q_{1}+1, q_{2}+1} e^{j 2 \pi \frac{u_{1}-k}{N} q_{1}} e^{-j 2 \pi \frac{u_{2}-m}{N} q_{2}}\right] \\
{[\mathcal{R}]_{k, m}(\mathrm{NDA})=\frac{\beta}{N^{2}} \sum_{q_{1}=0}^{N-1} \sum_{q_{2}=0}^{N-1}[\boldsymbol{\Gamma}]_{q_{1}+1, q_{2}+1} e^{j 2 \pi \frac{m q_{2}-k q_{1}}{N}} \sum_{u=1}^{N} e^{-j 2 \pi \frac{q_{1}-q_{2}}{N} u}=\frac{\beta}{N} \sum_{q=0}^{N-1}[\boldsymbol{\Gamma}]_{q+1, q+1} e^{j 2 \pi \frac{m-k}{N} q}} \\
{\left[\boldsymbol{\mathcal { R }}_{(n)}\right]_{k, m}(\mathrm{DA})=\frac{1}{N^{2}} \sum_{q_{1}=0}^{N-1} \sum_{q_{2}=0}^{N-1}[\boldsymbol{\Gamma}]_{q_{1}+1, q_{2}+1} e^{j 2 \pi \frac{m q_{2}-k q_{1}}{N}} \sum_{l=1}^{L} \sum_{u_{1}=1}^{N} \sum_{u_{2}=1}^{N} \sigma_{\alpha_{l}}^{2}\left[\mathbf{x}_{(n)}\right]_{u_{1}}\left[\mathbf{x}_{(n)}\right]_{u_{2}}^{*} e^{j 2 \pi \frac{q_{1}-\tau_{l}}{N} u_{1}} e^{-j 2 \pi \frac{q_{2}-\tau_{l}}{N} u_{2}}} \\
=\frac{1}{N^{2}} \sum_{q_{1}=0}^{N-1} \sum_{q_{2}=0}^{N-1}[\boldsymbol{\Gamma}]_{q_{1}+1, q_{2}+1}\left[\mathcal{Z}_{(n)}\right]_{q_{1}+1, q_{2}+1} e^{j 2 \pi} \frac{m q_{2}-k q_{1}}{N}
\end{gathered}
$$

as (59) where $\beta=\sum_{l=1}^{L} \sigma_{\alpha_{l}}^{2}$, and consequently we obtain the correlation matrix $\mathcal{R}$ in NDA context as defined by (28).

In DA context, the data symbols are known at the receiver then no averaging over the data is required. Hence, by using (58), the equation (57) becomes as (60) where the matrix $\mathcal{Z}_{(n)}$ is defined by (47), and consequently we obtain the correlation matrix $\mathcal{R}_{(n)}$ in DA context as defined by (46).

\section{APPENDIX B}

\section{EVALUation OF $\mathbf{J}_{m}$}

In this Appendix, we detail the calculus to obtain the expression of $\mathbf{J}_{m}$ defined in (35). Using the definition of $\mathcal{K}_{(n)}$ in section II, we have:

$$
\mathbf{A}=\mathcal{K}_{(n)}^{H} \boldsymbol{\Omega}^{-1} \mathcal{K}_{(n)}=\frac{1}{N^{2}}\left[\begin{array}{ccc}
\mathbf{A}_{1,1} & \cdots & \mathbf{A}_{1, L} \\
\vdots & \ddots & \vdots \\
\mathbf{A}_{L, 1} & \cdots & \mathbf{A}_{L, L}
\end{array}\right]
$$

where $\mathbf{A}_{l, l^{\prime}}=\mathbf{Z}_{l}^{(n)}{ }^{H} \boldsymbol{\Omega}^{-1} \mathbf{Z}_{l^{\prime}}^{(n)}$ is a $N_{c} \times N_{c}$ matrix with elements given by:

$$
\left[\mathbf{A}_{l, l^{\prime}}\right]_{d, d^{\prime}}=\mathbf{f}_{l}^{H} \operatorname{diag}\left\{\mathbf{x}_{(n)}^{H}\right\} \mathbf{M}_{d}^{H} \boldsymbol{\Omega}^{-1} \mathbf{M}_{d^{\prime}} \operatorname{diag}\left\{\mathbf{x}_{(n)}\right\} \mathbf{f}_{l^{\prime}}
$$


Taking the expectation of (63) over $\mathbf{x}$, we obtain:

$$
\mathrm{E}_{\mathbf{x}}\left[\left[\mathbf{A}_{l, l^{\prime}}\right]_{d, d^{\prime}}\right]=\mathbf{f}_{l}^{H} \mathcal{M}_{d, d^{\prime}} \mathbf{f}_{l^{\prime}}
$$

since the symbols are normalized an uncorrelated with respect to each other. Consequently, we obtain that:

$$
\mathrm{E}_{\mathbf{x}}\left[\mathbf{A}_{l, l^{\prime}}\right]=\mathcal{F}_{l}^{H} \mathcal{M}_{d, d^{\prime}} \mathcal{F}_{l^{\prime}}
$$

and finally we obtain the expression of $\mathbf{J}_{m}$ defined in (35).

\section{APPENDIX C}

\section{DERIVATION OF EXPRESSION (49) AND (50)}

Substituting (31) in (27), we obtain:

$$
\ln \left(p\left(\mathbf{y}_{(n)} \mid \mathbf{c}_{(n)}\right)\right)=-\frac{1}{\sigma^{2}}\left(\mathbf{y}_{(n)}^{H} \mathbf{y}_{(n)}+\mathbf{m}_{(n)}^{H} \mathbf{m}_{(n)}\right)+\ln \left(\frac{p\left(\mathbf{x}_{(n)}\right)}{\left|\pi \sigma^{2} \mathbf{I}_{N}\right|} \sum_{\mathbf{x}_{(n)}} e^{\frac{2}{\sigma^{2}} \operatorname{Re}\left(\mathbf{y}_{(n)}^{H} \mathbf{m}_{(n)}\right)}\right)
$$

since the normalized 4QAM-symbols are equiprobable (i.e., $\left.p\left(\mathbf{x}_{(n)}\right)=\frac{1}{4^{N}}\right)$. However, in this case $\mathbf{m}_{(n)}=$ $\operatorname{diag}\left\{\mathbf{x}_{(n)}\right\} \mathbf{F} \mathbf{c}_{(n)}$ then, $\mathbf{y}_{(n)}^{H} \mathbf{m}_{(n)}=\sum_{k=1}^{N} a_{n}(k)\left[\mathbf{x}_{(n)}\right]_{k}$ where $a_{n}(k)$ is defined in section IV part B. Hence, we obtain:

$$
\sum_{\mathbf{x}_{(n)}} e^{\frac{2}{\sigma^{2}} \operatorname{Re}\left(\mathbf{y}_{(n)}^{H} \mathbf{m}_{(n)}\right)}=\prod_{k=1}^{N}\left(\sum_{\left[\mathbf{x}_{(n)}\right]_{k}} e^{\frac{2}{\sigma^{2}} \operatorname{Re}\left(a_{n}(k)\left[\mathbf{x}_{(n)}\right]_{k}\right)}\right)
$$

Since $\left[\mathbf{x}_{(n)}\right]_{k}=\frac{1}{\sqrt{2}}( \pm 1 \pm j)$ (i.e., 4QAM-symbol) then, we obtain:

$$
\sum_{\left[\mathbf{x}_{(n)}\right]_{k}} e^{\frac{2}{\sigma^{2}} \operatorname{Re}\left(a_{n}(k)\left[\mathbf{x}_{(n)}\right]_{k}\right)}=4 \cosh \left(\frac{\sqrt{2}}{\sigma^{2}} \operatorname{Re}\left(a_{n}(k)\right)\right) \cosh \left(\frac{\sqrt{2}}{\sigma^{2}} \operatorname{Im}\left(a_{n}(k)\right)\right)
$$

Inserting this result into (66), we obtain the expression in (49). Taking the second derivative of (49) with respect to $\mathbf{c}_{(n)}$ and using these results below:

$$
\begin{aligned}
\nabla_{\mathbf{c}_{(n)}} \operatorname{Re}\left(a_{n}(k)\right) & =\frac{1}{2}\left[\mathbf{y}_{(n)}\right]_{k}^{*} \mathbf{g}_{k} \\
\nabla_{\mathbf{c}_{(n)}} \operatorname{Im}\left(a_{n}(k)\right) & =\frac{1}{2 j}\left[\mathbf{y}_{(n)}\right]_{k}^{*} \mathbf{g}_{k}
\end{aligned}
$$

we obtain finally the expression in (50). 


\section{APPENDIX D}

\section{EVALUATION OF $\mathbf{J}_{l}$}

In this Appendix, we detail the calculus to obtain the expression of $\mathbf{J}_{l}$ defined in (53). Inserting the definition of $a_{n}(k)$ into (52) and substituting the result in (26), we obtain:

$$
\mathbf{J}_{l}=\frac{1}{\sigma^{2}} \mathbf{F}^{H} \mathbf{F}-\frac{1}{\sigma^{4}} \sum_{k=1}^{N} \mathbf{g}_{k}^{*} \mathrm{E}_{\mathbf{c}} \mathrm{E}_{\mathbf{y} \mid \mathbf{c}}\left[\left[\mathbf{y}_{(n)}\right]_{k}\left[\mathbf{y}_{(n)}\right]_{k}^{*}\right] \mathbf{g}_{k}^{T}+\frac{1}{\sigma^{8}} \sum_{k=1}^{N} \mathbf{g}_{k}^{*} \mathbf{g}_{k}^{T} \mathrm{E}_{\mathbf{c}}\left[\mathbf{c}_{(n)} \mathbf{c}_{(n)}^{H} \mathbf{g}_{k}^{*} \mathrm{E}_{\mathbf{y} \mid \mathbf{c}}\left[\left(\left[\mathbf{y}_{(n)}\right]_{k}\left[\mathbf{y}_{(n)}\right]_{k}^{*}\right)^{2}\right]\right] \mathbf{g}_{k}^{T}
$$

Using that $\left[\mathbf{y}_{(n)}\right]_{k}=\left[\mathbf{x}_{(n)}\right]_{k} \mathbf{g}_{k}^{T} \mathbf{c}_{(n)}+\left[\mathbf{w}_{(n)}\right]_{k}$, the normalized symbols and the noise are independant and, these results below:

$$
\begin{aligned}
& \mathrm{E}_{\left[\mathbf{x}_{(n)}\right]_{k}}\left[\left[\mathbf{x}_{(n)}\right]_{k}^{2}\right]=\mathrm{E}_{\left[\mathbf{w}_{(n)}\right]_{k}}\left[\left[\mathbf{w}_{(n)}\right]_{k}^{2}\right]=0 \\
& \mathrm{E}_{\left[\mathbf{w}_{(n)}\right]_{k}}\left[\left[\mathbf{w}_{(n)}\right]_{k}^{2}\left[\mathbf{w}_{(n)}\right]_{k}^{* 2}\right]=2 \sigma^{4}
\end{aligned}
$$

we obtain:

$$
\begin{aligned}
\mathrm{E}_{\mathbf{y} \mid \mathbf{c}}\left[\left[\mathbf{y}_{(n)}\right]_{k}\left[\mathbf{y}_{(n)}\right]_{k}^{*}\right] & =\mathbf{g}_{k}^{T} \mathbf{c}_{(n)} \mathbf{c}_{(n)}^{H} \mathbf{g}_{k}^{*}+\sigma^{2} \\
\mathrm{E}_{\mathbf{y} \mid \mathbf{c}}\left[\left(\left[\mathbf{y}_{(n)}\right]_{k}\left[\mathbf{y}_{(n)}\right]_{k}^{*}\right)^{2}\right] & =2 \sigma^{4}+4 \sigma^{2} \mathbf{g}_{k}^{T} \mathbf{c}_{(n)} \mathbf{c}_{(n)}^{H} \mathbf{g}_{k}^{*}+\mathbf{g}_{k}^{T} \mathbf{c}_{(n)} \mathbf{c}_{(n)}^{H} \mathbf{g}_{k}^{*} \mathbf{g}_{k}^{T} \mathbf{c}_{(n)} \mathbf{c}_{(n)}^{H} \mathbf{g}_{k}^{*}
\end{aligned}
$$

Hence, $\mathbf{J}_{l}$ becomes:

$$
\begin{aligned}
\mathbf{J}_{l}= & \frac{1}{\sigma^{4}} \sum_{k=1}^{N} \mathbf{V}_{k} \mathrm{E}_{\mathbf{c}}\left[\mathbf{c}_{(n)} \mathbf{c}_{(n)}^{H}\right] \mathbf{V}_{k} \\
& +\frac{4}{\sigma^{6}} \sum_{k=1}^{N} \mathbf{V}_{k} \mathrm{E}_{\mathbf{c}}\left[\mathbf{c}_{(n)} \mathbf{c}_{(n)}^{H} \mathbf{V}_{k} \mathbf{c}_{(n)} \mathbf{c}_{(n)}^{H}\right] \mathbf{V}_{k} \\
& +\frac{1}{\sigma^{8}} \sum_{k=1}^{N} \mathbf{V}_{k} \mathrm{E}_{\mathbf{c}}\left[\mathbf{c}_{(n)} \mathbf{c}_{(n)}^{H} \mathbf{V}_{k} \mathbf{c}_{(n)} \mathbf{c}_{(n)}^{H} \mathbf{V}_{k} \mathbf{c}_{(n)} \mathbf{c}_{(n)}^{H}\right] \mathbf{V}_{k}
\end{aligned}
$$

where $\mathbf{V}_{k}=\mathbf{g}_{k}^{*} \mathbf{g}_{k}^{T}$ is a $L \times L$ matrix. Let $\mathbf{T}_{1}=\mathbf{c}_{(n)} \mathbf{c}_{(n)}^{H} \mathbf{V}_{k} \mathbf{c}_{(n)} \mathbf{c}_{(n)}^{H}$ and $\mathbf{T}_{2}=\mathbf{c}_{(n)} \mathbf{c}_{(n)}^{H} \mathbf{V}_{k} \mathbf{c}_{(n)} \mathbf{c}_{(n)}^{H} \mathbf{V}_{k} \mathbf{c}_{(n)} \mathbf{c}_{(n)}^{H}$ then, the elements of these matrices are given by:

$$
\begin{aligned}
& {\left[\mathbf{T}_{1}\right]_{l, l^{\prime}}=\sum_{l_{1}=1}^{L} \sum_{l_{2}=1}^{L}\left[\mathbf{V}_{k}\right]_{l 1, l 2}\left[\mathbf{c}_{(n)}\right]_{l}\left[\mathbf{c}_{(n)}\right]_{l_{2}}\left[\mathbf{c}_{(n)}\right]_{l^{\prime}}^{*}\left[\mathbf{c}_{(n)}\right]_{l_{1}}^{*}} \\
& {\left[\mathbf{T}_{2}\right]_{l, l^{\prime}}=\sum_{l_{1}=1}^{L} \sum_{l_{2}=1}^{L} \sum_{l_{3}=1}^{L} \sum_{l_{4}=1}^{L}\left[\mathbf{V}_{k}\right]_{l 1, l 2}\left[\mathbf{V}_{k}\right]_{l 3, l 4}\left[\mathbf{c}_{(n)}\right]_{l}\left[\mathbf{c}_{(n)}\right]_{l_{2}}\left[\mathbf{c}_{(n)}\right]_{l_{4}}\left[\mathbf{c}_{(n)}\right]_{l^{\prime}}^{*}\left[\mathbf{c}_{(n)}\right]_{l_{1}}^{*}\left[\mathbf{c}_{(n)}\right]_{l_{3}}^{*}}
\end{aligned}
$$

Using that $\mathbf{D}=\mathrm{E}_{\mathbf{c}}\left[\mathbf{c}_{(n)} \mathbf{c}_{(n)}^{H}\right]=\operatorname{diag}\left\{\sigma_{\alpha_{1}}^{2}, \ldots, \sigma_{\alpha_{L}}^{2}\right\}, \mathrm{E}_{\left[\mathbf{c}_{(n)}\right]_{l}}\left[\left[\mathbf{c}_{(n)}\right]_{l}^{2}\right]=0$ and the definitions of fourth and sixth order moments for complex Gaussian variables, we obtain:

$$
\begin{aligned}
& \mathrm{E}_{\mathbf{c}}\left[\mathbf{T}_{1}\right]=\mathbf{D} \mathbf{V}_{k} \mathbf{D}+\operatorname{Tr}\left(\mathbf{V}_{k} \mathbf{D}\right) \mathbf{D} \\
& \mathrm{E}_{\mathbf{c}}\left[\mathbf{T}_{2}\right]=2 \mathbf{D V}_{k} \mathbf{D} \mathbf{V}_{k} \mathbf{D}+2 \operatorname{Tr}\left(\mathbf{V}_{k} \mathbf{D}\right) \mathbf{D} \mathbf{V}_{k} \mathbf{D}+\operatorname{Tr}\left(\mathbf{V}_{k} \mathbf{D} \mathbf{V}_{k} \mathbf{D}\right) \mathbf{D}+\left(\operatorname{Tr}\left(\mathbf{V}_{k} \mathbf{D}\right)\right)^{2} \mathbf{D}
\end{aligned}
$$


Using that $\mathbf{g}_{k}^{T} \mathbf{D} \mathbf{g}_{k}^{*}=\operatorname{Tr}\left(\mathbf{V}_{k} \mathbf{D}\right)=\sum_{l=1}^{L} \sigma_{\alpha_{l}}^{2}=\beta, \operatorname{Tr}\left(\mathbf{V}_{k} \mathbf{D} \mathbf{V}_{k} \mathbf{D}\right)=\beta^{2}$ and $\mathbf{D V}_{k} \mathbf{D} \mathbf{V}_{k} \mathbf{D}=\beta \mathbf{D V} \mathbf{V}_{k} \mathbf{D}$ and, inserting these results into (73), we obtain finally the expression of $\mathbf{J}_{l}$ defined in (53).

\section{APPENDIX E}

\section{PROOF OF THE INEQUALITY (55)}

From the definition of $\mathbf{J}_{\min }$, we have $\mathbf{J}_{\min } \leq \mathbf{J}_{m}=\mathbf{J}_{h}$ and then we have the first inequality in (55), i.e., $\operatorname{MBCRB}(\mathbf{c}) \leq \mathbf{A B C R B}(\mathbf{c})$. To prove the second inequality in (55), we have to show that $\mathbf{J} \leq \mathbf{J}_{\min }$. From Fig. 8, we see that the $\tanh ^{2}(x)$ function is tangent to the curve $y=x^{2}$ at $x=0$ and has $y=1$ as horizontal asymptote. Hence, we can write, for every $x \geq 0$, these two properties below:

$$
\tanh ^{2}(x) \leq 1 \text { and } \tanh ^{2}(x) \leq x^{2}
$$

Using these two properties, we obtain from (50) that:

$$
\begin{aligned}
& \Delta_{\mathbf{c}_{(n)}}^{\mathbf{c}_{(n)}} \ln \left(p\left(\mathbf{y}_{(n)} \mid \mathbf{c}_{(n)}\right)\right) \geq-\frac{1}{\sigma^{2}} \mathbf{F}^{H} \mathbf{F} \\
& \Delta_{\mathbf{c}_{(n)}}^{\mathbf{c}_{(n)}} \ln \left(p\left(\mathbf{y}_{(n)} \mid \mathbf{c}_{(n)}\right)\right) \geq-\frac{1}{\sigma^{2}} \mathbf{F}^{H} \mathbf{F}+\sum_{k=1}^{N}\left[\frac{1}{\sigma^{8}}\left[\mathbf{y}_{(n)}\right]_{k}\left[\mathbf{y}_{(n)}\right]_{k}^{*} \mathbf{g}_{k}^{*} \mathbf{g}_{k}^{T}\left(\sigma^{4}-a_{n}(k) a_{n}^{*}(k)\right)\right]
\end{aligned}
$$

Substituting (77) and (78) in (26), we obtain:

$$
\mathbf{J} \leq \mathbf{J}_{h} \text { and } \mathbf{J} \leq \mathbf{J}_{l}
$$

Hence, we have $\mathbf{J} \leq \mathbf{J}_{\min }$, and consequently the second inequality in (55), i.e., $\mathbf{A B C R B}(\mathbf{c}) \leq \mathbf{B C R B}(\mathbf{c})$.

\section{REFERENCES}

[1] H. Hijazi and L. Ros, “ On-line Bayesian Cramer-Rao Bounds for OFDM Slowly Varying Rayleigh Multi-path Channel Estimation” in IEEE ISWCS Conf., Reykjavik, Iceland, October 2008.

[2] H. Hijazi and L. Ros, “ OFDM High Speed Channel Complex Gains Estimation Using Kalman Filter and QR-Detector” in IEEE ISWCS Conf., Reykjavik, Iceland, October 2008.

[3] H. Hijazi and L. Ros, " Polynomial estimation of time-varying multi-path gains with intercarrier interference mitigation in OFDM systems” in IEEE Trans. Vehic. Techno., Vol. 57, No. 6, November 2008.

[4] H. Hijazi and L. Ros, " Polynomial Estimation of Time-varying Multi-path Gains with ICI Mitigation in OFDM Systems" in IEEE ISCCSP Conf., St. Julians, MALTA, March 2008.

[5] H. Hijazi and L. Ros, “ Time-varying Channel Complex Gains Estimation and ICI Suppression in OFDM Systems” in IEEE Global Communications Conf., Washington, USA, Nov. 2007.

[6] M. K. Tsatsanis and G. B. Giannakis, "Subspace Methods for Blind Estimation of Time-Varying FIR Channels " in IEEE Trans. Signal Process., vol. 45, no. 12, pp. 3084-3093, Dec. 1997.

[7] D. K. Borah and B. D. Hart, "Frequency-Selective Fading Channel Estimation with a Polynomial Time-Varying Channel Model” in IEEE Trans. Commun., Vol. 47, No. 6, pp. 862-873, June 1999. 
[8] X. Wang and H. J. R. Liu, "An adaptive channel estimation algorithm using time-frequency polynomial model for OFDM with multi-path channels" in EURASIP Journal on Applied Signal Processing, pp. 818-830, Aug. 2002.

[9] H. Senol, H. A. Cirpan and E. Panayirci, "A low-complexity KL expansion-based channel estimator for OFDM systems" in EURASIP Journal on Wireless Communications and Networking, pp. 163-174, Feb. 2005.

[10] Z. Tang, R. C. Cannizzaro, G. Leus and P. Banelli, "Pilot-assisted time-varying channel estimation for OFDM systems" in IEEE Trans. Signal Process., vol. 55, pp. 2226-2238, May 2007.

[11] S. Tomasin, A. Gorokhov, H. Yang and J.-P. Linnartz, "Iterative interference cancellation and channel estimation for mobile OFDM" in IEEE Trans. Wireless Commun., vol. 4, no. 1, pp. 238-245, Jan. 2005.

[12] T. Zemen and C. F. Mecklenbrauker, "Time-Variant Channel Estimation Using Discrete Prolate Spheroidal Sequences" in IEEE Trans. Signal Process., vol. 53, no. 9, pp. 3597-3607, Sep. 2005.

[13] Y. Mostofi and D. Cox, "ICI mitigation for pilot-aided OFDM mobile systems" in IEEE Trans. Wireless Commun., vol. 4, no. 12, pp. 765-774, March 2005.

[14] B. Yang, K. B. Letaief, R. S. Cheng and Z. Cao, "Channel estimation for OFDM transmisson in mutipath fading channels based on parametric channel modeling" in IEEE Trans. Commun., vol. 49, no. 3, pp. 467-479, March 2001.

[15] E. Simon, L. Ros and K. Raoof,“ Synchronization over rapidly time-varying multipath channel for CDMA downlink RAKE receivers in Time-Division mode”, in IEEE Trans. Vehic. Techno., vol. 56. no. 4, Jul. 2007

[16] E. Simon and L. Ros,“ Adaptive multipath channel estimation in CDMA based on prefiltering and combination with a linear equalizer",14th IST Mobile and Wireless Communications Summit, Dresden, June 2005.

[17] W. C. Jakes, Microwave Mobile Communications. IEEE Press, 1983.

[18] Wikipedia contributors, "Linear regression", Wikipedia, The Free Encyclopedia.

[19] A. R. S. Bahai and B. R. Saltzberg, Multi-Carrier Dications: Theory and Applications of OFDM: Kluwer Academic/Plenum, 1999.

[20] H. L. Van Trees, Detection, estimation, and modulation theory: Part I, Wiley, New York, 1968.

[21] A. D’Andrea, U. Mengali and R. Reggiannini, “ The modified Cramer-Rao bound and its application to synchronization problems" in IEEE Trans. Commun., vol. 42, pp. 1391-1399, Apr. 1994.

[22] F. Gini, R. Reggiannini and U. Mengali, “ The modified Cramer-Rao bound in vector parameter estimation " in IEEE Trans. Commun., vol. 46, pp. 52-60, Jan. 1998.

[23] B. Z. Bobrovsky, E. Mayer-Wolf and M. Zakai, “ Some classes of global Cramer-Rao bounds” in Ann. Statis., vol. 15, pp. 1421-1438, 1987.

[24] P. Tichavsky, C. H. Murachvik and A. Nehorai, " Posterior Cramer-Rao bound for discret-time nonlinear filtering” in IEEE Trans. Signal Processing, vol. 46, pp. 1386-1396, May. 1998.

[25] S. Bay, C. Herzet, J. M. Brossier, J. P. Barbot and B. Geller, “Analytic and asymptotic analysis of Bayesian Cramer-Rao bound for dynamical phase offset estimation” in IEEE Trans. Signal Processing, vol. 56, pp. 61-70, Jan. 2008.

[26] M. Moeneclaey, "On the true and the modified Cramer-Rao bounds for the estimation of a scalar parameter in the presence of nuisance parameters" in IEEE Trans. Commun., vol. 46, no. 11, pp. 1536-1544, Nov. 1998.

[27] Steven M. Kay, Fundamentals of Statistical Signal Processing: Estimation Theory, Prentice Hall PTR, 1993.

[28] R. L. Peterson, R. E. Ziemer and D. E. Borth, Introduction of Spread Spectrum Communications, Prentice Hall Inc., 1995. 
TABLE I

The Minimum of the BCRB $(\alpha)$ For GSM Channel [3]

\begin{tabular}{|c|c|c|c|c|}
\hline SNR $(\mathrm{dB})$ & 0 & & & 40 \\
\hline 0.05 & $N_{c}=2$ & $N_{c}=2$ & $N_{c}=2$ & $N_{c}=2$ \\
\hline 0.1 & $N_{c}=3$ & $N_{c}=3$ & $N_{c}=3$ & $N_{c}=3$ \\
\hline 0.2 & $N_{c}=3$ & $N_{c}=3$ & $N_{c}=3$ & $N_{c}=4$ \\
\hline 0.3 & $N_{c}=3$ & $N_{c}=3$ & $N_{c}=4$ & $N_{c}=4$ \\
\hline 0.4 & $N_{c}=3$ & $N_{c}=4$ & $N_{c}=4$ & $N_{c}=4$ \\
\hline
\end{tabular}

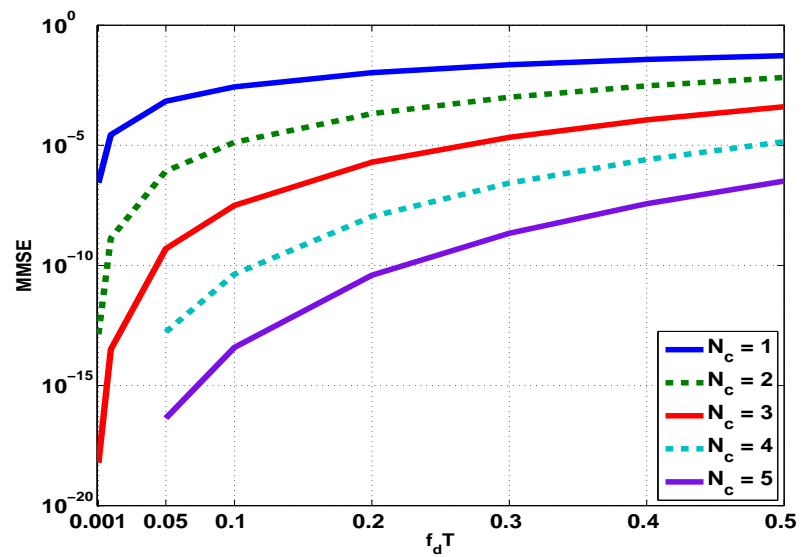

Fig. 1. MMSE for a normalized channel with $L=6$ paths and $v=144$

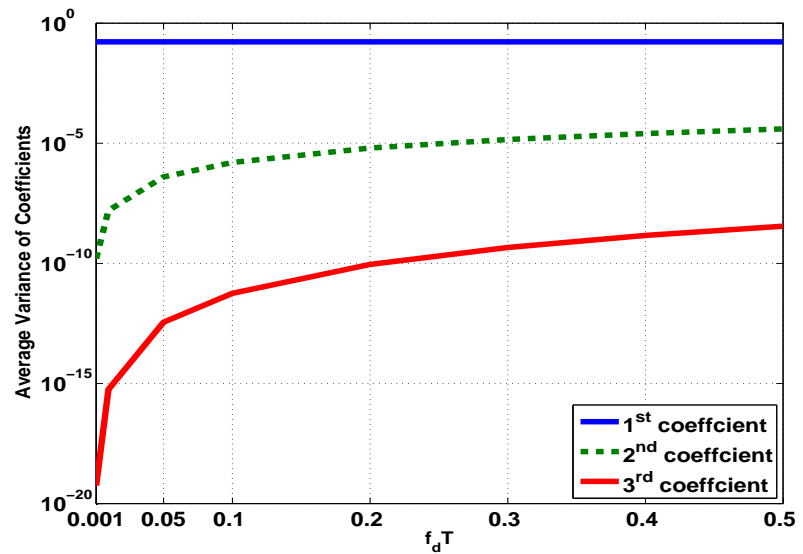

Fig. 2. Average variances of the first three coefficients for a normalized channel with $L=6$ paths and $v=144$ 


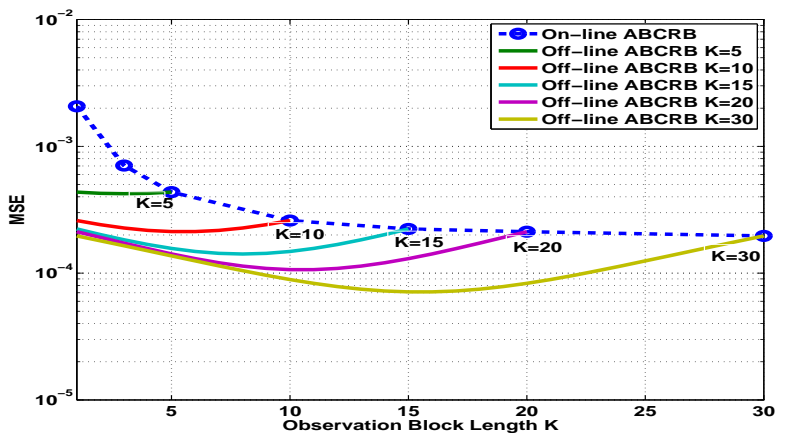

(a)

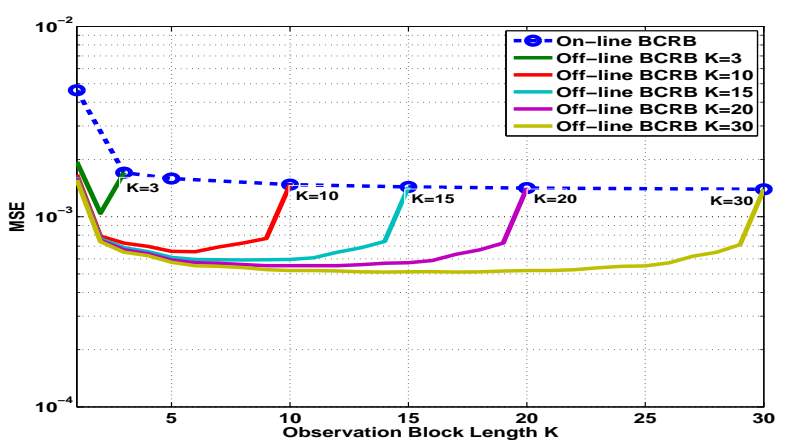

(b)

Fig. 3. BCRBs vs number of observations, for SNR $=10 d B$ : (a) time-invariant with $f_{d} T=0.001$; (b) time-varying with $f_{d} T=0.1$ and $N_{c}=2$ 


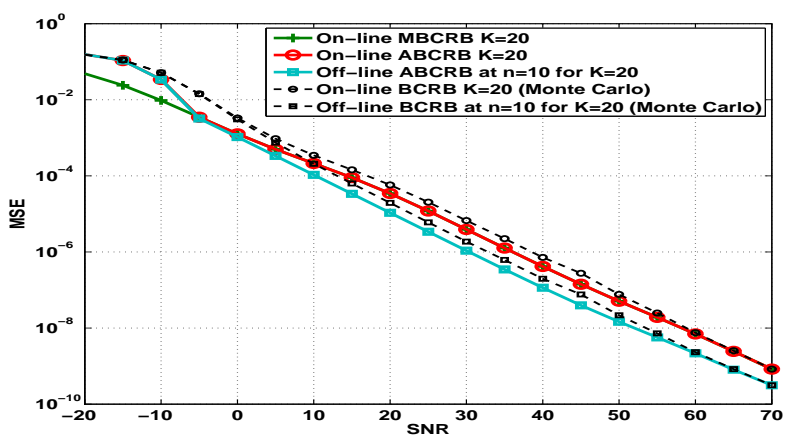

(a)

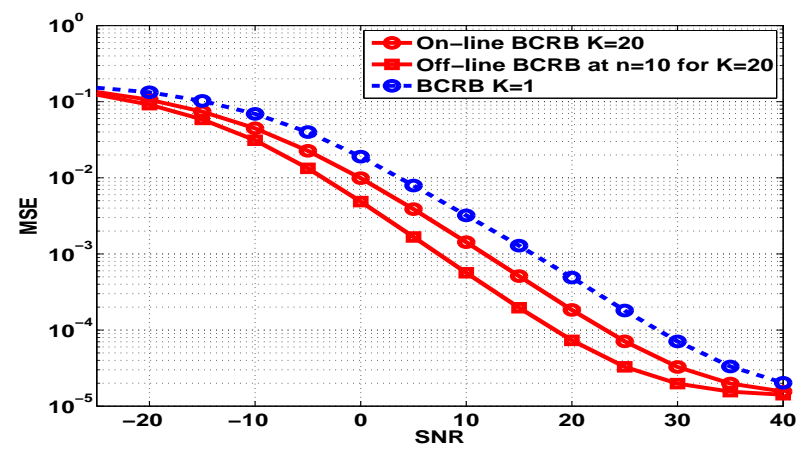

(b)

Fig. 4. BCRBs vs SNR: (a) time-invariant with $f_{d} T=0.001$ ( $\mathbf{J}$ is evaluated over $10^{4}$ OFDM symbols Monte Carlo trials); (b) DA time-varying with $f_{d} T=0.1$ and $N_{c}=2$ 


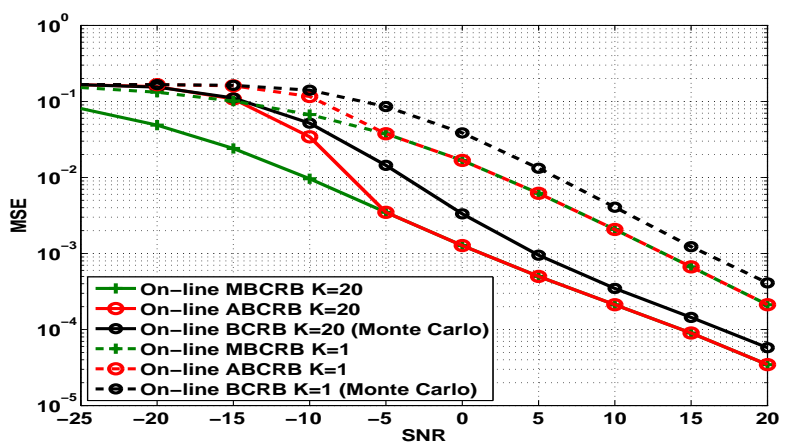

(a)

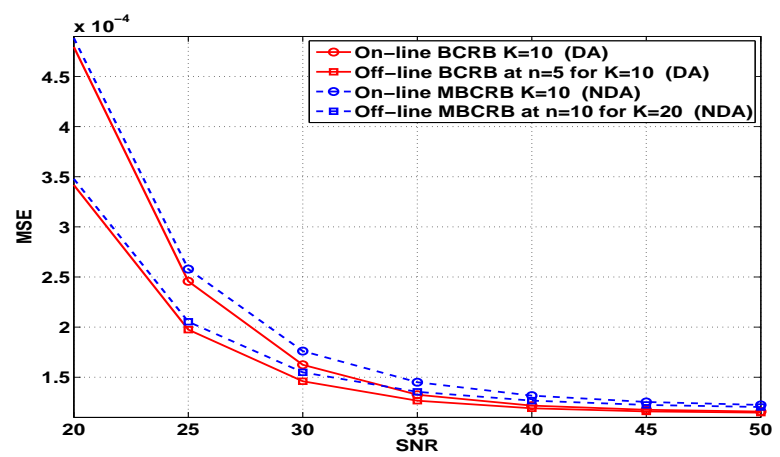

(b)

Fig. 5. BCRBs vs SNR in DA and NDA contexts: (a) time-invariant with $f_{d} T=10^{-3}$ and two different observation lengths $K=1$ and $K=20$; (b) time-varying with $f_{d} T=0.4, N_{c}=3$ and $K=10$ 


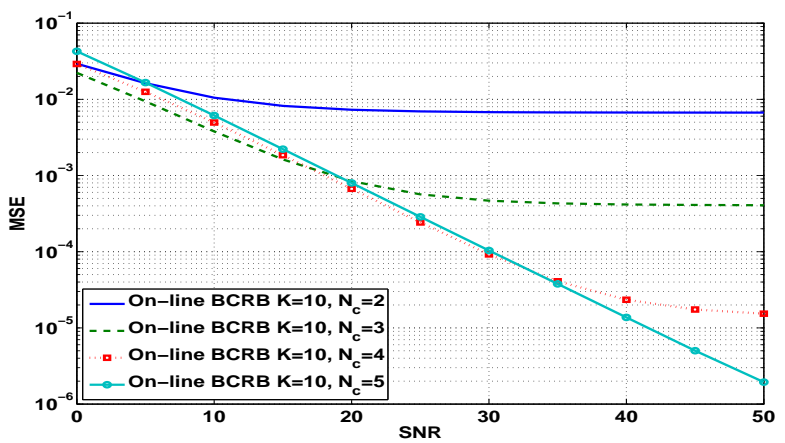

(a)

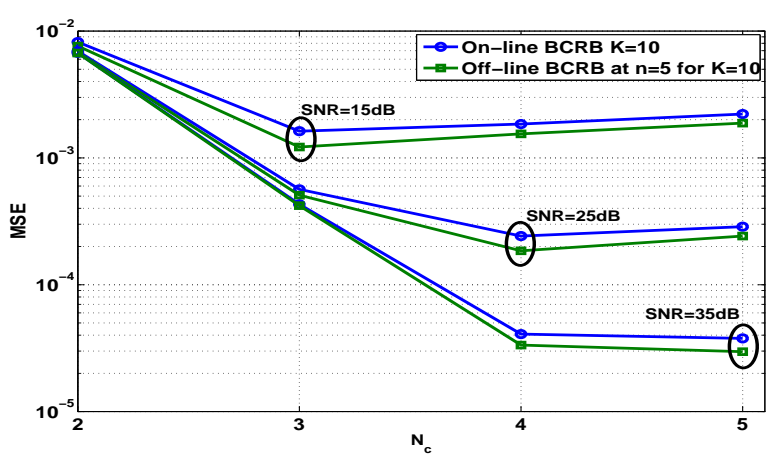

(b)

Fig. 6. (a) BCRBs vs SNR for $f_{d} T=0.5$ and $N_{c}=2$ to 5 ; (b) BCRBs vs $N_{c}$ for $f_{d} T=0.5$ and $\mathrm{SNR}=15 d B, 25 \mathrm{~dB}$ and $35 \mathrm{~dB}$

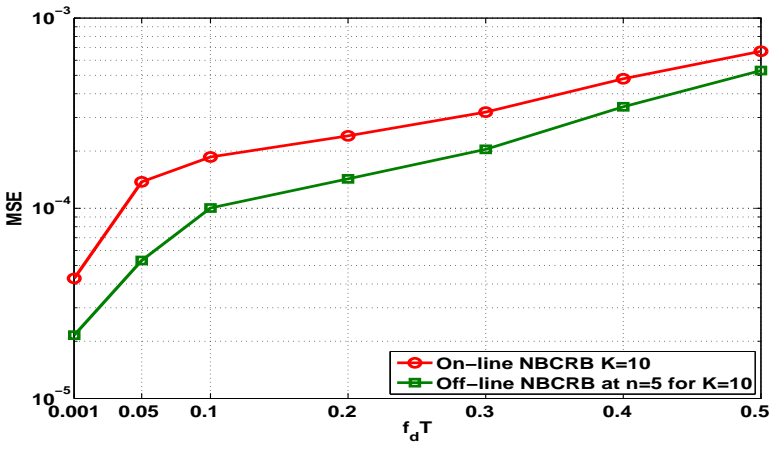

Fig. 7. NBCRBs vs $f_{d} T$ for $\mathrm{SNR}=20 d B$ 


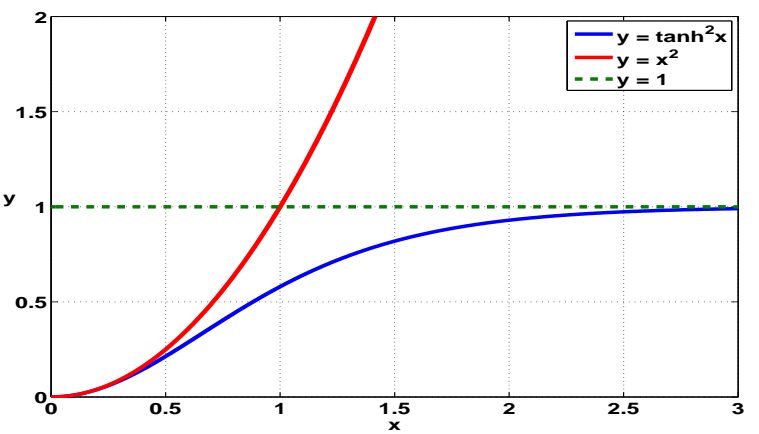

Fig. 8. the $\tanh ^{2}(\cdot)$ function 